\title{
A fourth-order linearized difference scheme for the coupled space fractional Ginzburg-Landau equation
}

\author{
Yuan $\mathrm{Xu}^{1}$, Jiali Zeng ${ }^{1}$ and Shuanggui $\mathrm{Hu}^{1,2^{*}}$
}

\section{"Correspondence:}

hushuanggui808@csu.edu.cn 'School of Mathematics and Statistics, Central South University, Changsha, China

${ }^{2}$ School of Geosciences and Info-Physics, Central South University, Changsha, China

\begin{abstract}
In this paper, the coupled space fractional Ginzburg-Landau equations are investigated numerically. A linearized semi-implicit difference scheme is proposed. The scheme is unconditionally stable, fourth-order accurate in space, and second-order accurate in time. The optimal pointwise error estimates, unique solvability, and unconditional stability are obtained. Moreover, Richardson extrapolation is exploited to improve the temporal accuracy to fourth order. Finally, numerical results are presented to confirm the theoretical results.
\end{abstract}

Keywords: Ginzburg-Landau equation; Fractional Laplacian; Pointwise error estimate; Unconditional stability; Fourth-order convergence

\section{Introduction}

The Ginzburg-Landau equation (GLE) has been used to model a wide variety of physical systems [1]. The fractional Ginzburg-Landau equation (FGLE) was first suggested by Tarasov and Zaslavsky [2,3] for fractal media. The FGLE has been exploited to describe many physical situations $[2,4,5]$, where the FGLE is characterized by the fractional power of the Laplacian. Recently, the coupled fractional Ginzburg-Landau equation (CFGLE) with stochastic noise was discussed by Shu et al. [6].

There are quite a lot of numerical studies for the classical GLE and FGLE, see [7-15] and the references therein. To the authors' best knowledge, there are not too many numerical studies for the CFGLE. As far as we are aware, only very recently, Li and Huang [16] proposed a second-order implicit midpoint scheme for CFGLE. However, the method in [16] is a nonlinear scheme which requires some iterations at each time step, and only $L^{2}$-norm error estimates are provided.

In this paper, we consider the following CFGLE:

$$
\begin{aligned}
u_{t} & +\left(v_{1}+i \eta_{1}\right)(-\Delta)^{\frac{\alpha}{2}} u \\
& +\left(\left(\kappa_{1}+i \zeta_{1}\right)|u|^{2}+\left(\delta_{1}+i \beta_{1}\right)|v|^{2}\right) u-\gamma_{1} u=0, \quad x \in \mathbb{R}, 0<t<T, \\
v_{t} & +\left(v_{2}+i \eta_{2}\right)(-\Delta)^{\frac{\alpha}{2}} v \\
& +\left(\left(\kappa_{2}+i \zeta_{2}\right)|u|^{2}+\left(\delta_{2}+i \beta_{2}\right)|v|^{2}\right) v-\gamma_{2} v=0, \quad x \in \mathbb{R}, 0<t<T,
\end{aligned}
$$

(0) The Author(s) 2019. This article is distributed under the terms of the Creative Commons Attribution 4.0 International License (http://creativecommons.org/licenses/by/4.0/), which permits unrestricted use, distribution, and reproduction in any medium, provided you give appropriate credit to the original author(s) and the source, provide a link to the Creative Commons license, and indicate if changes were made. 
with the initial condition

$$
u(x, 0)=u_{0}(x), \quad v(x, 0)=v_{0}(x), \quad x \in \mathbb{R},
$$

where $i=\sqrt{-1}$ is the complex unit, $u(x, t), v(x, t)$ are complex-valued functions of time variable $t$ and space variable $x, v_{1}>0, v_{2}>0, \kappa_{1}, \kappa_{2}, \delta_{1}, \delta_{2}, \eta_{1}, \eta_{2}, \zeta_{1}, \zeta_{2}, \gamma_{1}, \gamma_{2}, \beta_{1}, \beta_{2}$ are given real constants, $u_{0}(x), v_{0}(x)$ are complex-valued functions satisfying certain regularity, and $1<\alpha \leq 2$. The fractional Laplacian can be regarded as the Riesz fractional derivative $[17-22]$

$$
(-\Delta)^{\frac{\alpha}{2}} f(x)=-\partial_{x}^{\alpha} f(x)=\frac{1}{2 \cos (\alpha \pi / 2)}\left(-\infty D_{x}^{\alpha}+{ }_{x} D_{+\infty}^{\alpha}\right) f(x),
$$

where ${ }_{-\infty} D_{x}^{\alpha} f(x)$ denotes the left Riemann-Liouville fractional derivative

$$
{ }_{-\infty} D_{x}^{\alpha} f(x)=\frac{1}{\Gamma(2-\alpha)} \frac{d^{2}}{d x^{2}} \int_{-\infty}^{x} \frac{f(\xi)}{(x-\xi)^{\alpha-1}} d \xi
$$

and ${ }_{x} D_{+\infty}^{\alpha} f(x)$ denotes the right Riemann-Liouville fractional derivative

$$
{ }_{x} D_{+\infty}^{\alpha} f(x)=\frac{1}{\Gamma(2-\alpha)} \frac{d^{2}}{d x^{2}} \int_{x}^{+\infty} \frac{f(\xi)}{(\xi-x)^{\alpha-1}} d \xi
$$

If $v_{1}=\kappa_{1}=\delta_{1}=\gamma_{1}=v_{2}=\kappa_{2}=\delta_{2}=\gamma_{2}=0$, Eqs. (1)-(2) reduce to the coupled nonlinear Schrödinger equation [19].

The objective of this paper is to develop an unconditionally stable linearized scheme with optimal pointwise error estimates for the above CFGLE. The method, which uses three time levels, is shown to be fourth-order convergence in space variable and secondorder convergence in time variable in the sense of $L^{\infty}$-norm. Moreover, Richardson extrapolation is exploited to improve the temporal accuracy to fourth order. And the method is also shown to be almost unconditionally stable (the time step is not related to the spatial meshsize).

The rest of this paper is organized as follows. Section 2 gives the linearized implicit finite difference method. Section 3 provides the theoretical analysis for the proposed scheme, which includes the convergence and stability. Section 4 presents the numerical results which confirm the theoretical results. And the conclusion is given in the final section.

\section{A three-level linearized implicit difference scheme}

\subsection{Spatial discretization}

In this paper, we adopt the fourth-order central difference scheme proposed by [17] to the spatial discretization for the Riesz fractional derivative, where the method is based on the Ortigueira's second-order scheme and an average operator [17, 23].

Lemma 1 (see [23, 24]) For $\alpha>-1$, the fractional centered difference is defined by

$$
\Delta_{h}^{\alpha} f(x)=\frac{1}{h^{\alpha}} \sum_{k=-\infty}^{\infty} c_{k}^{\alpha} f(x-k h)
$$


where

$$
c_{k}^{\alpha}=\frac{(-1)^{k} \Gamma(\alpha+1)}{\Gamma\left(\frac{\alpha}{2}-k+1\right) \Gamma\left(\frac{\alpha}{2}+k+1\right)}=\left(1-\frac{\alpha+1}{\frac{\alpha}{2}+k}\right) c_{k-1}^{\alpha} \quad \text { for } k \in \mathbb{Z} .
$$

The coefficients $c_{k}^{\alpha}$ have the following properties:

$$
c_{0}^{\alpha}>0, \quad c_{k}^{\alpha}=c_{-k}^{\alpha} \leq 0 \quad \text { for } k= \pm 1, \pm 2, \ldots
$$

Lemma 2 (see [24]) Let $f(x) \in C^{5}(\mathbb{R}) \cap L^{1}(\mathbb{R})$ and all spatial derivatives of $f(x)$ up to order five belong to $L^{1}(\mathbb{R})$. Then

$$
(-\Delta)^{\frac{\alpha}{2}} f(x)=\Delta_{h}^{\alpha} f(x)+O\left(h^{2}\right)
$$

for $1<\alpha \leq 2$.

Lemma 3 ([17]) Let $f \in C^{7}(\mathbb{R}) \cap L^{1}(\mathbb{R})$ and all derivatives up to order seven belong to $L^{1}(\mathbb{R})$ and $1<\alpha \leq 2$, then

$$
\frac{1}{h^{\alpha}} \sum_{k=-\infty}^{\infty} c_{k}^{\alpha} f(x-k h)=\mathcal{A}_{x}^{\alpha}\left((-\Delta)^{\frac{\alpha}{2}} f(x)\right)+O\left(h^{4}\right),
$$

where the average operator $\mathcal{A}_{h}^{\alpha}$ is defined as

$$
\mathcal{A}_{x}^{\alpha} f(x)=\frac{\alpha}{24} f(x-h)+\left(1-\frac{\alpha}{12}\right) f(x)+\frac{\alpha}{24} f(x+h) .
$$

\subsection{Fractional Sobolev norm}

Under certain conditions, the solution of problem (1)-(3) converges to zero when $|x| \rightarrow$ $\infty$. Thus, in practical numerical computation, we truncate the original problem on a bounded interval and take the following homogeneous Dirichlet boundary conditions:

$$
u(x, t)=0, \quad v(x, t)=0, \quad x \in \mathbb{R} /(a, b), t \in[0, T],
$$

where $a$ and $b$ are usually chosen sufficiently large negative and positive numbers.

In the numerical computation, the solution domain is defined as $\{(x, t) \mid a \leq x \leq b, 0 \leq$ $t \leq T\}$, which is covered by a uniform grid $\left\{\left(x_{j}, t_{n}\right) \mid x_{j}=a+j h, t_{n}=n \tau, j=0, \ldots, M, n=\right.$ $0, \ldots, N\}$, with spacing $h=\frac{b-a}{M}, \tau=\frac{T}{N}$, where $M, N$ are two positive integers. For any grid function $P^{n}=\left\{P_{j}^{n}\right\}(0 \leq j \leq M)$, the following notations are introduced:

$$
P_{j}^{\bar{n}}=\frac{P_{j}^{n+1}+P_{j}^{n-1}}{2}, \quad \delta_{t} P_{j}^{n}=\frac{P_{j}^{n+1}-P_{j}^{n-1}}{2 \tau} .
$$

Denote

$$
Z_{h}^{0}=\left\{P \mid P=\left\{P_{j}\right\}, P_{0}=P_{M}=0\right\} .
$$


For any grid functions $P, Q \in Z_{h}^{0}$, we define the discrete inner product and the associated $L^{2}$-norm

$$
(P, Q)=h \sum_{j=1}^{M-1} P_{j} \bar{Q}_{j}, \quad\|P\|=\sqrt{(P, P)} .
$$

The discrete $L^{\infty}$-norm is defined as

$$
\|P\|_{\infty}=\max _{1 \leq j \leq M-1}\left|P_{j}\right| .
$$

Let $L_{h}^{2}=\left\{P \mid P \in Z_{h}^{0},\|P\|<+\infty\right\}$, and for any given $\delta \in[0,1]$, the fractional Sobolev norm $\|P\|_{H^{\delta}}$ and seminorm $|P|_{H^{\delta}}$ are defined as

$$
\|P\|_{H^{\delta}}^{2}=\int_{-\pi / h}^{\pi / h}\left(1+|k|^{2 \delta}\right)|\hat{P}(k)|^{2} d k, \quad|P|_{H^{\delta}}^{2}=\int_{-\pi / h}^{\pi / h}|k|^{2 \delta}|\hat{P}(k)|^{2} d k
$$

where the relation between the semi-discrete Fourier transform $\hat{P}(k)$ and the grid function is given by

$$
\hat{P}(k)=\frac{h}{\sqrt{2 \pi}} \sum_{1 \leq j \leq M-1} P_{j} e^{-i k x_{j}}, \quad S_{j}=\frac{1}{\sqrt{2 \pi}} \int_{-\pi / h}^{\pi / h} \hat{P}(k) e^{i k x_{j}} d k .
$$

Obviously, $\|P\|_{H^{\delta}}^{2}=\|P\|^{2}+|P|_{H^{\delta}}^{2}$. Let $H_{h}^{\delta}:=\left\{P \in Z_{h}^{0} \mid\|P\|_{H^{\delta}}<+\infty\right\}$, then we introduce the following two lemmas which are shown in [25].

Lemma 4 ([25]) For any $1<\alpha \leq 2$ and $P \in H_{h}^{\frac{\alpha}{2}}$, there exists a constant $C_{\alpha}>0$, independent of $h$, such that

$$
\|P\|_{\infty} \leq C_{\alpha}\|P\|_{H^{\frac{\alpha}{2}}}
$$

Lemma 5 ([25]) For every $1<\alpha \leq 2$, we have

$$
\left(\frac{2}{\pi}\right)^{\alpha}|P|_{H^{\frac{\alpha}{2}}}^{2} \leq\left(\Delta_{h}^{\alpha} P, P\right) \leq|P|_{H^{\frac{\alpha}{2}}}^{2}, \quad \forall P \in H_{h}^{\frac{\alpha}{2}}
$$

and

$$
\left(\frac{2}{\pi}\right)^{\alpha}|P|_{H^{\frac{\alpha}{2}}}|Q|_{H^{\frac{\alpha}{2}}} \leq \sum_{j \in Z}\left|\Delta_{h}^{\alpha} P_{j} \bar{Q}_{j}\right| \leq|P|_{H^{\frac{\alpha}{2}}}|Q|_{H^{\frac{\alpha}{2}}}, \quad \forall P, Q \in H_{h}^{\frac{\alpha}{2}} .
$$

With the assumption of homogenous boundary condition (12), for any $P \in Z_{h}^{0}$, we have

$$
\Delta_{h}^{\alpha} P_{j}=\frac{1}{h^{\alpha}} \sum_{k=j-1}^{j-M+1} c_{k}^{\alpha} P_{j-k}=\frac{1}{h^{\alpha}} \sum_{k=1}^{M-1} c_{j-k}^{\alpha} P_{k} .
$$




\subsection{Numerical scheme}

A three-level linearized difference scheme [26-29] for boundary value problem (1)-(3) with homogeneous Dirichlet boundary conditions (12) in the finite domain $\Omega=[a, b]$ is as follows:

$$
\begin{aligned}
& \mathcal{A}_{x}^{\alpha} \delta_{t} U_{j}^{n}+\left(v_{1}+i \eta_{1}\right) \Delta_{h}^{\alpha} U_{j}^{\bar{n}}+\mathcal{A}_{x}^{\alpha}\left[\left(\left(\kappa_{1}+i \zeta_{1}\right)\left|U_{j}^{n}\right|^{2}+\left(\delta_{1}+i \beta_{1}\right)\left|V_{j}^{n}\right|^{2}\right) U_{j}^{\bar{n}}\right]-\gamma_{1} \mathcal{A}_{x}^{\alpha} U_{j}^{\bar{n}} \\
& \quad=0, \quad 0<j<M, 1<n<N, \\
& \mathcal{A}_{x}^{\alpha} \delta_{t} V_{j}^{n}+\left(v_{2}+i \eta_{2}\right) \Delta_{h}^{\alpha} V_{j}^{\bar{n}}+\mathcal{A}_{x}^{\alpha}\left[\left(\left(\kappa_{2}+i \zeta_{2}\right)\left|U_{j}^{n}\right|^{2}+\left(\delta_{2}+i \beta_{2}\right)\left|V_{j}^{n}\right|^{2}\right) V_{j}^{\bar{n}}\right]-\gamma_{2} \mathcal{A}_{x}^{\alpha} V_{j}^{\bar{n}} \\
& =0, \quad 0<j<M, 1<n<N, \\
& U_{j}^{0}=u_{0}\left(x_{j}\right), \quad V_{j}^{0}=v_{0}\left(x_{j}\right), \quad 0<j<M, \\
& U_{0}^{n}=U_{M}^{n}=0, \quad V_{0}^{n}=V_{M}^{n}=0, \quad 0 \leq n \leq N .
\end{aligned}
$$

Since the difference scheme involves three time levels, the first step values $U_{j}^{1}, V_{j}^{1}$ are required to begin stepping the numerical solution forward in time.

Using Taylor expansion and Eqs. (1) and (2), one has

$$
\begin{aligned}
u^{1}=u_{0} & +\tau u_{t}(x, 0)+O\left(\tau^{2}\right) \\
=u_{0} & -\tau\left(\left(v_{1}+i \eta_{1}\right)(-\Delta)^{\frac{\alpha}{2}} u_{0}\right. \\
& \left.+\left(\left(\kappa_{1}+i \zeta_{1}\right)\left|u_{0}\right|^{2}+\left(\delta_{1}+i \beta_{1}\right)\left|v_{0}\right|^{2}\right) u_{0}-\gamma_{1} u_{0}\right)+O\left(\tau^{2}\right), \\
v^{1}=v_{0} & +\tau v_{t}(x, 0)+O\left(\tau^{2}\right) \\
=v_{0} & -\tau\left(\left(v_{2}+i \eta_{2}\right)(-\Delta)^{\frac{\alpha}{2}} v_{0}\right. \\
& \left.+\left(\left(\kappa_{2}+i \zeta_{2}\right)\left|u_{0}\right|^{2}+\left(\delta_{2}+i \beta_{2}\right)\left|v_{0}\right|^{2}\right) v_{0}-\gamma_{2} v_{0}\right)+O\left(\tau^{2}\right) .
\end{aligned}
$$

In addition, from Lemma 2 we know

$$
\begin{aligned}
& (-\Delta)^{\frac{\alpha}{2}} u_{0}\left(x_{j}\right)=\frac{1}{h^{\alpha}} \sum_{k=1}^{M-1} c_{j-k}^{\alpha} u_{0}\left(x_{j}\right)+O\left(h^{2}\right)=\Delta_{h}^{\alpha} u_{0}\left(x_{j}\right)+O\left(h^{2}\right), \\
& (-\Delta)^{\frac{\alpha}{2}} v_{0}\left(x_{j}\right)=\frac{1}{h^{\alpha}} \sum_{k=1}^{M-1} c_{j-k}^{\alpha} v_{0}\left(x_{j}\right)+O\left(h^{2}\right)=\Delta_{h}^{\alpha} v_{0}\left(x_{j}\right)+O\left(h^{2}\right) .
\end{aligned}
$$

In the numerical simulation, $U^{1}, V^{1}$ are obtained from the following scheme:

$$
\begin{aligned}
U_{j}^{1}= & U_{j}^{0}-\tau\left(\left(v_{1}+i \eta_{1}\right) \Delta_{h}^{\alpha} U_{j}^{0}\right. \\
& \left.+\left(\left(\kappa_{1}+i \zeta_{1}\right)\left|U_{j}^{0}\right|^{2}+\left(\delta_{1}+i \beta_{1}\right)\left|V_{j}^{0}\right|^{2}\right) U_{j}^{0}-\gamma_{1} U_{j}^{0}\right), \quad 0<j<M, \\
V_{j}^{1}= & V_{j}^{0}-\tau\left(\left(v_{2}+i \eta_{2}\right) \Delta_{h}^{\alpha} V_{j}^{0}\right. \\
& \left.+\left(\left(\kappa_{2}+i \zeta_{2}\right)\left|U_{j}^{0}\right|^{2}+\left(\delta_{2}+i \beta_{2}\right)\left|V_{j}^{0}\right|^{2}\right) V_{j}^{0}-\gamma_{2} V_{j}^{0}\right), \quad 0<j<M .
\end{aligned}
$$




\section{Theoretical analysis}

Lemma 6 For any grid functions $P, Q \in Z_{h}^{0}$, we have [17]

$$
\left(\mathcal{A}_{x}^{\alpha} P, Q\right)=\left(P, \mathcal{A}_{x}^{\alpha} Q\right)
$$

Moreover,

$$
\left\|\mathcal{A}_{x}^{\alpha} P\right\| \leq\|P\|, \quad \frac{1}{3}\|P\|^{2} \leq\|P\|_{A}^{2} \leq\|P\|^{2},
$$

where $\|P\|_{A}=\sqrt{\left(\mathcal{A}_{x}^{\alpha} P, P\right)}$.

Lemma 7 For any two grid functions $P, Q \in Z_{h}^{0}$, there exists a linear operator $\Lambda^{\alpha}$ such that [30]

$$
\left(\Delta_{h}^{\alpha} P, Q\right)=\left(\Lambda^{\alpha} P, \Lambda^{\alpha} Q\right) .
$$

Lemma 8 For any grid functions $P^{n} \in Z_{h}^{0}$, we have

$$
\begin{aligned}
& \operatorname{Im}\left(\Delta_{h}^{\alpha} P^{n}, P^{n}\right)=0, \\
& \operatorname{Re}\left(\mathcal{A}_{x}^{\alpha} \delta_{t} P^{n}, P^{\bar{n}}\right)=\frac{1}{4 \tau}\left(\left\|P^{n+1}\right\|_{A}^{2}-\left\|P^{n-1}\right\|_{A}^{2}\right), \\
& \operatorname{Re}\left(\mathcal{A}_{x}^{\alpha} \delta_{t} P^{n}, \Delta_{h}^{\alpha} P^{\bar{n}}\right)=\frac{1}{4 \tau}\left(\left\|\Lambda^{\alpha} P^{n+1}\right\|_{A}^{2}-\left\|\Lambda^{\alpha} P^{n-1}\right\|_{A}^{2}\right) .
\end{aligned}
$$

Lemma 9 (Discrete Gronwall's inequality [31,32]) Let $\left\{u_{k}\right\}$ and $\left\{w_{k}\right\}$ be nonnegative sequences and $\alpha$ be a nonnegative constant satisfying

$$
u_{n} \leq \alpha+\sum_{0 \leq k<n} w_{k} u_{k} \text { for } n \geq 0
$$

Then, for all $n$, it holds

$$
u_{n} \leq \alpha \exp \left(\sum_{0 \leq k<n} w_{k}\right)
$$

\section{1 $L^{\infty}$ convergence}

Let $u(x, t), v(x, t)$ be the exact solution of problem (1)-(3) and (12), $U_{j}^{n}, V_{j}^{n}$ be the solution of numerical schemes (22)-(25). Let $u_{j}^{n}=u\left(x_{j}, t_{n}\right), v_{j}^{n}=v\left(x_{j}, t_{n}\right)$, the error functions

$$
e_{j}^{n}=u_{j}^{n}-U_{j}^{n}, \quad \xi_{j}^{n}=v_{j}^{n}-V_{j}^{n}, \quad j=1,2, \ldots, M, n=1,2, \ldots, N .
$$

Define the truncation errors of scheme (22)-(23) as follows:

$$
\begin{aligned}
r_{j}^{n}= & \mathcal{A}_{x}^{\alpha} \delta_{t} u_{j}^{n}+\left(v_{1}+i \eta_{1}\right) \Delta_{h}^{\alpha} u_{j}^{\bar{n}} \\
& +\mathcal{A}_{x}^{\alpha}\left[\left(\left(\kappa_{1}+i \zeta_{1}\right)\left|u_{j}^{n}\right|^{2}+\left(\delta_{1}+i \beta_{1}\right)\left|v_{j}^{n}\right|^{2}\right) u_{j}^{\bar{n}}\right]-\gamma_{1} \mathcal{A}_{x}^{\alpha} u_{j}^{\bar{n}}, \\
s_{j}^{n}= & \mathcal{A}_{x}^{\alpha} \delta_{t} v_{j}^{n}+\left(v_{2}+i \eta_{2}\right) \Delta_{h}^{\alpha} v_{j}^{\bar{n}} \\
& +\mathcal{A}_{x}^{\alpha}\left[\left(\left(\kappa_{2}+i \zeta_{2}\right)\left|u_{j}^{n}\right|^{2}+\left(\delta_{2}+i \beta_{2}\right)\left|v_{j}^{n}\right|^{2}\right) v_{j}^{\bar{n}}\right]-\gamma_{2} \mathcal{A}_{x}^{\alpha} v_{j}^{\bar{n}},
\end{aligned}
$$


for $1 \leq j \leq M-1$ and $1 \leq n \leq N-1$.

Subtracting (22) from (40) and subtracting (23) from (41) yield that

$$
\begin{aligned}
& r_{j}^{n}=\mathcal{A}_{x}^{\alpha} \delta_{t} e_{j}^{n}+\left(v_{1}+i \eta_{1}\right) \Delta_{h}^{\alpha} e_{j}^{\bar{n}}+\mathcal{A}_{x}^{\alpha} P_{j}^{n}-\gamma_{1} \mathcal{A}_{x}^{\alpha} e_{j}^{\bar{n}}, \\
& s_{j}^{n}=\mathcal{A}_{x}^{\alpha} \delta_{t} \xi_{j}^{n}+\left(v_{2}+i \eta_{2}\right) \Delta_{h}^{\alpha} \xi_{j}^{\bar{n}}+\mathcal{A}_{x}^{\alpha} Q_{j}^{n}-\gamma_{2} \mathcal{A}_{x}^{\alpha} \xi_{j}^{\bar{n}},
\end{aligned}
$$

where

$$
\begin{aligned}
& P_{j}^{n}=\left(\left(\kappa_{1}+i \zeta_{1}\right)\left|u_{j}^{n}\right|^{2}+\left(\delta_{1}+i \beta_{1}\right)\left|v_{j}^{n}\right|^{2}\right) u_{j}^{\bar{n}}-\left(\left(\kappa_{1}+i \zeta_{1}\right)\left|U_{j}^{n}\right|^{2}+\left(\delta_{1}+i \beta_{1}\right)\left|V_{j}^{n}\right|^{2}\right) U_{j}^{\bar{n}}, \\
& Q_{j}^{n}=\left(\left(\kappa_{2}+i \zeta_{2}\right)\left|u_{j}^{n}\right|^{2}+\left(\delta_{2}+i \beta_{2}\right)\left|v_{j}^{n}\right|^{2}\right) v_{j}^{\bar{n}}-\left(\left(\kappa_{2}+i \zeta_{2}\right)\left|U_{j}^{n}\right|^{2}+\left(\delta_{2}+i \beta_{2}\right)\left|V_{j}^{n}\right|^{2}\right) V_{j}^{\bar{n}} .
\end{aligned}
$$

Using Taylor expansion and Lemma 3, we can easily obtain the following lemma.

Lemma 10 Suppose that the solution of problem (1)-(3) is sufficiently smooth. Then it holds that

$$
\left|r_{j}^{n}\right| \leq C_{R}\left(\tau^{2}+h^{4}\right), \quad\left|s_{j}^{n}\right| \leq C_{R}\left(\tau^{2}+h^{4}\right), \quad 1 \leq j \leq M-1,1 \leq n \leq N-1,
$$

where $C_{R}$ is a positive constant independent of $\tau$ and $h$.

Following a similar proof of Lemma 9 in [14], we can obtain the lemma below.

Lemma 11 Suppose that the solution of problem (1)-(3) is sufficiently smooth. Then one has

$$
\begin{array}{ll}
\left|e_{j}^{1}\right| \leq C_{e}\left(\tau^{2}+\tau h^{2}\right), & \left|\Delta_{h}^{\alpha} e_{j}^{1}\right| \leq C_{e}\left(\tau^{2}+\tau h^{2+\alpha}\right), \\
\left|\xi_{j}^{1}\right| \leq C_{e}\left(\tau^{2}+\tau h^{2}\right), & \left|\Delta_{h}^{\alpha} \xi_{j}^{1}\right| \leq C_{e}\left(\tau^{2}+\tau h^{2+\alpha}\right),
\end{array}
$$

where $C_{e}$ is a positive constant independent of $\tau$ and $h$.

Theorem 1 Suppose that the solution of problem (1)-(3) is smooth enough, then there exist two small positive constants $\tau_{0}$ and $h_{0}$ such that, when $\tau<\tau_{0}$ and $h<h_{0}$, the numerical solution $\left(U^{n}, V^{n}\right)$ of difference schemes (22)-(25) and (30)-(31) converges to the exact solution $\left(u^{n}, v^{n}\right)$ in the sense of $L^{\infty}$-norm with the optimal convergence order $O\left(\tau^{2}+h^{4}\right)$, i.e.,

$$
\left\|u^{n}-U^{n}\right\|_{\infty} \leq C_{0}\left(\tau^{2}+h^{4}\right), \quad\left\|v^{n}-V^{n}\right\|_{\infty} \leq C_{0}\left(\tau^{2}+h^{4}\right), \quad 1 \leq n \leq N
$$

where $C_{0}$ is a positive constant independent of $\tau$ and $h$.

Proof We use mathematical induction to prove (46). It follows from (45) that the error estimate (46) holds for $n=1$ when $h \leq 1$. Indeed,

$$
\left\|u^{1}-U^{1}\right\|_{\infty} \leq \frac{5}{4} C_{e}\left(\tau^{2}+h^{4}\right)
$$

when $h \leq 1$, and $\tau h^{2} \leq \frac{\tau^{2}}{4}+h^{4}$ is used. 
Now assume that (46) is valid for $m \leq n$, we want to show that (46) is also valid for $n+1$. By the assumption, one has

$$
\begin{aligned}
& \left\|U^{m}\right\|_{\infty} \leq\left\|u^{m}\right\|_{\infty}+\left\|e^{m}\right\|_{\infty} \leq C_{m}+C_{0}\left(\tau^{2}+h^{4}\right) \leq C_{m}+1, \quad 1 \leq m \leq n, \\
& \left\|V^{m}\right\|_{\infty} \leq\left\|v^{m}\right\|_{\infty}+\left\|\xi^{m}\right\|_{\infty} \leq C_{m}+C_{0}\left(\tau^{2}+h^{4}\right) \leq C_{m}+1, \quad 1 \leq m \leq n,
\end{aligned}
$$

for $\tau<\tau_{1}$ and $h<h_{1}$, where $\tau_{1}, h_{1}$ satisfy that $\tau_{1}^{2}+h_{1}^{4}<\frac{1}{C_{0}}$. Here,

$$
C_{m}=\max \left\{\max _{a \leq x \leq b, 0 \leq t \leq T}|u(x, t)|, \max _{a \leq x \leq b, 0 \leq t \leq T}|v(x, t)|\right\} .
$$

Now, computing the discrete inner product of (42) with $e^{\bar{n}}$ and taking the real part of the resulting equation, we have

$$
\frac{\left\|e^{n+1}\right\|_{A}^{2}-\left\|e^{n-1}\right\|_{A}^{2}}{4 \tau}+v_{1}\left\|\Lambda^{\alpha} e^{\bar{n}}\right\|^{2}=-\operatorname{Re}\left[\left(\mathcal{A}_{x}^{\alpha} P^{n}, e^{\bar{n}}\right)\right]+\gamma_{1}\left\|e^{\bar{n}}\right\|_{A}^{2}+\operatorname{Re}\left[\left(r^{n}, e^{\bar{n}}\right)\right]
$$

where

$$
\begin{aligned}
P_{j}^{n}= & \left(\kappa_{1}+i \zeta_{1}\right)\left|u_{j}^{n}\right|^{2} u_{j}^{\bar{n}}-\left(\kappa_{1}+i \zeta_{1}\right)\left|U_{j}^{n}\right|^{2} U_{j}^{\bar{n}}+\left(\delta_{1}+i \beta_{1}\right)\left|v_{j}^{n}\right|^{2} u_{j}^{\bar{n}}-\left(\delta_{1}+i \beta_{1}\right)\left|V_{j}^{n}\right|^{2} U_{j}^{\bar{n}} \\
= & \left(\kappa_{1}+i \zeta_{1}\right)\left(\left(\left|u_{j}^{n}\right|^{2}-\left|U_{j}^{n}\right|^{2}\right) u_{j}^{\bar{n}}+\left|U_{j}^{n}\right|^{2} e_{j}^{\bar{n}}\right)+\left(\delta_{1}+i \beta_{1}\right)\left(\left(\left|v_{j}^{n}\right|^{2}-\left|V_{j}^{n}\right|^{2}\right) u_{j}^{\bar{n}}+\left|V_{j}^{n}\right|^{2} e_{j}^{\bar{n}}\right) \\
= & \left(\kappa_{1}+i \zeta_{1}\right)\left(\left(\left|u_{j}^{n}\right|-\left|U_{j}^{n}\right|\right)\left(\left|u_{j}^{n}\right|+\left|U_{j}^{n}\right|\right) u_{j}^{\bar{n}}+\left|U_{j}^{n}\right|^{2} e_{j}^{\bar{n}}\right) \\
& +\left(\delta_{1}+i \beta_{1}\right)\left(\left(\left|v_{j}^{n}\right|-\left|V_{j}^{n}\right|\right)\left(\left|v_{j}^{n}\right|+\left|V_{j}^{n}\right|\right) u_{j}^{\bar{n}}+\left|V_{j}^{n}\right|^{2} e_{j}^{\bar{n}}\right) .
\end{aligned}
$$

By using assumption (47), one has

$$
\begin{aligned}
\left\|P^{n}\right\| \leq & \sqrt{\kappa_{1}^{2}+\zeta_{1}^{2}}\left(\left(\left\|u^{n}\right\|_{\infty}+\left\|U^{n}\right\|_{\infty}\right)\left\|u^{\bar{n}}\right\|_{\infty}\left\|e^{n}\right\|+\left\|U^{n}\right\|_{\infty}^{2}\left\|e^{\bar{n}}\right\|\right) \\
& +\sqrt{\delta_{1}^{2}+\zeta_{1}^{2}}\left(\left(\left\|v^{n}\right\|_{\infty}+\left\|V^{n}\right\|_{\infty}\right)\left\|u^{\bar{n}}\right\|_{\infty}\left\|\xi^{n}\right\|+\left\|V^{n}\right\|_{\infty}^{2}\left\|e^{\bar{n}}\right\|\right) \\
\leq & \sqrt{\kappa_{1}^{2}+\zeta_{1}^{2}}\left(\left(C_{m}+C_{m}+1\right) C_{m}\left\|e^{n}\right\|+\left(C_{m}+1\right)^{2}\left\|e^{\bar{n}}\right\|\right) \\
& +\sqrt{\delta_{1}^{2}+\zeta_{1}^{2}}\left(\left(C_{m}+C_{m}+1\right) C_{m}\left\|\xi^{n}\right\|+\left(C_{m}+1\right)^{2}\left\|e^{\bar{n}}\right\|\right) \\
\leq & C_{1}\left(\left\|e^{n}\right\|+\left\|\xi^{n}\right\|+\left\|e^{\bar{n}}\right\|\right)
\end{aligned}
$$

for $\tau<\tau_{1}$ and $h<h_{1}$, where

$$
C_{1}=2\left(C_{m}+1\right)^{2} \max \left\{\sqrt{\kappa_{1}^{2}+\zeta_{1}^{2}}, \sqrt{\delta_{1}^{2}+\beta_{1}^{2}}\right\} .
$$

Thus,

$$
\left\|P^{n}\right\|^{2} \leq 3 C_{1}^{2}\left(\left\|e^{n}\right\|^{2}+\left\|\xi^{n}\right\|^{2}+\left\|e^{\bar{n}}\right\|^{2}\right) .
$$

\section{Moreover,}

$$
\left|-\operatorname{Re}\left[\left(\mathcal{A}_{x}^{\alpha} P^{n}, e^{\bar{n}}\right)\right]\right| \leq\left|\left(\mathcal{A}_{x}^{\alpha} P^{n}, e^{\bar{n}}\right)\right|
$$




$$
\begin{aligned}
& \leq\left\|\mathcal{A}_{x}^{\alpha} P^{n}\right\|\left\|e^{\bar{n}}\right\| \\
& \leq \frac{1}{2}\left(\left\|\mathcal{A}_{x}^{\alpha} P^{n}\right\|^{2}+\left\|e^{\bar{n}}\right\|^{2}\right) \\
& \leq \frac{1}{2}\left(\left\|P^{n}\right\|^{2}+\left\|e^{\bar{n}}\right\|^{2}\right) \\
& \leq \frac{1}{2}\left(3 C_{1}^{2}\left(\left\|e^{n}\right\|^{2}+\left\|\xi^{n}\right\|^{2}+\left\|e^{\bar{n}}\right\|^{2}\right)+\left\|e^{\bar{n}}\right\|^{2}\right) \\
& \leq \frac{3 C_{1}^{2}+1}{2}\left(\left\|e^{n}\right\|^{2}+\left\|\xi^{n}\right\|^{2}+2\left\|e^{\bar{n}}\right\|^{2}\right) \\
& \leq \frac{3 C_{1}^{2}+1}{2}\left(\left\|e^{n-1}\right\|^{2}+\left\|e^{n}\right\|^{2}+\left\|e^{n+1}\right\|^{2}+\left\|\xi^{n}\right\|^{2}\right) \\
& \leq \frac{3\left(3 C_{1}^{2}+1\right)}{2}\left(\left\|e^{n-1}\right\|_{A}^{2}+\left\|e^{n}\right\|_{A}^{2}+\left\|e^{n+1}\right\|_{A}^{2}+\left\|\xi^{n}\right\|_{A}^{2}\right)
\end{aligned}
$$

where Lemma 6 is used.

Similarly, one has

$$
\begin{aligned}
\left|\operatorname{Re}\left(r^{n}, e^{\bar{n}}\right)\right| & \leq \frac{1}{2}\left(\left\|r^{n}\right\|^{2}+\left\|e^{\bar{n}}\right\|^{2}\right) \\
& \leq \frac{1}{2}\left(\left\|r^{n}\right\|^{2}+\left\|e^{n+1}\right\|^{2}+\left\|e^{n-1}\right\|^{2}\right) \\
& \leq \frac{1}{2}\left\|r^{n}\right\|^{2}+\frac{3}{2}\left(\left\|e^{n+1}\right\|_{A}^{2}+\left\|e^{n-1}\right\|_{A}^{2}\right),
\end{aligned}
$$

where Lemma 6 is used.

From (48), (52), and (53), we have

$$
\begin{aligned}
& \frac{\left\|e^{n+1}\right\|_{A}^{2}-\left\|e^{n-1}\right\|_{A}^{2}}{4 \tau} \\
& =-v_{1}\left\|\Lambda^{\alpha} e^{\bar{n}}\right\|^{2}-\operatorname{Re}\left[\left(\mathcal{A}_{x}^{\alpha} P^{n}, e^{\bar{n}}\right)\right]+\gamma_{1}\left\|e^{\bar{n}}\right\|_{A}^{2}+\operatorname{Re}\left[\left(r^{n}, e^{\bar{n}}\right)\right] \\
& \leq \frac{3\left(3 C_{1}^{2}+1\right)}{2}\left(\left\|e^{n-1}\right\|_{A}^{2}+\left\|e^{n}\right\|_{A}^{2}+\left\|e^{n+1}\right\|_{A}^{2}+\left\|\xi^{n}\right\|_{A}^{2}\right) \\
& \quad+\frac{\left|\gamma_{1}\right|}{2}\left(\left\|e^{n+1}\right\|_{A}^{2}+\left\|e^{n-1}\right\|_{A}^{2}\right)+\frac{1}{2}\left\|r^{n}\right\|^{2}+\frac{3}{2}\left(\left\|e^{n+1}\right\|_{A}^{2}+\left\|e^{n-1}\right\|_{A}^{2}\right) \\
& \leq C_{2}\left(\left\|e^{n+1}\right\|_{A}^{2}+\left\|e^{n}\right\|_{A}^{2}+\left\|e^{n-1}\right\|_{A}^{2}+\left\|\xi^{n}\right\|_{A}^{2}\right) \\
& \quad+\frac{\left|\gamma_{1}\right|}{2}\left(\left\|e^{n+1}\right\|_{A}^{2}+\left\|e^{n-1}\right\|_{A}^{2}\right)+\frac{1}{2}\left\|r^{n}\right\|^{2},
\end{aligned}
$$

where $C_{2}=\frac{9 C_{1}^{2}+6}{2}$. Thus,

$$
\begin{aligned}
\left\|e^{n+1}\right\|_{A}^{2}-\left\|e^{n-1}\right\|_{A}^{2} \leq & 4 C_{2} \tau\left(\left\|e^{n+1}\right\|_{A}^{2}+\left\|e^{n}\right\|_{A}^{2}+\left\|e^{n-1}\right\|_{A}^{2}+\left\|\xi^{n}\right\|_{A}^{2}\right)+2 \tau\left\|r^{n}\right\|^{2} \\
& +2\left|\gamma_{1}\right| \tau\left(\left\|e^{n+1}\right\|_{A}^{2}+\left\|e^{n-1}\right\|_{A}^{2}\right) .
\end{aligned}
$$


Similarly, computing the discrete inner product of (43) with $\xi^{\bar{n}}$ and analyzing the resulting equation, one can obtain

$$
\begin{aligned}
\left\|\xi^{n+1}\right\|_{A}^{2}-\left\|\xi^{n-1}\right\|_{A}^{2} \leq & 4 C_{2}^{\prime} \tau\left(\left\|\xi^{n+1}\right\|_{A}^{2}+\left\|\xi^{n}\right\|_{A}^{2}+\left\|\xi^{n-1}\right\|_{A}^{2}+\left\|e^{n}\right\|_{A}^{2}\right)+2 \tau\left\|s^{n}\right\|^{2} \\
& +2\left|\gamma_{2}\right| \tau\left(\left\|\xi^{n+1}\right\|_{A}^{2}+\left\|\xi^{n-1}\right\|_{A}^{2}\right),
\end{aligned}
$$

where $C_{2}^{\prime}=\frac{9\left(C_{1}^{\prime}\right)^{2}+6}{2}$, and

$$
C_{1}^{\prime}=2\left(C_{m}+1\right)^{2} \max \left\{\sqrt{\kappa_{2}^{2}+\zeta_{2}^{2}}, \sqrt{\delta_{2}^{2}+\beta_{2}^{2}}\right\}
$$

Next, computing the discrete inner product of (42) with $\Delta_{h}^{\alpha} e^{\bar{n}}$ and taking the real part of the resulting equation, we obtain

$$
\begin{aligned}
& \frac{\left\|\Lambda^{\alpha} e^{n+1}\right\|_{A}^{2}-\left\|\Lambda^{\alpha} e^{n-1}\right\|_{A}^{2}}{4 \tau}+v_{1}\left\|\Delta_{h}^{\alpha} e^{\bar{n}}\right\|^{2} \\
& \quad=\operatorname{Re}\left[-\left(\mathcal{A}_{x}^{\alpha} P^{n}, \Delta_{h}^{\alpha} e^{\bar{n}}\right)\right]+\gamma_{1}\left\|\Lambda^{\alpha} e^{\bar{n}}\right\|_{A}^{2}+\operatorname{Re}\left(r^{n}, \Delta_{h}^{\alpha} e^{\bar{n}}\right),
\end{aligned}
$$

where Lemma 6 and Lemma 7 are used.

From (50), one obtains

$$
\left\|P^{n}\right\|^{2} \leq 3 C_{1}^{2}\left(\left\|e^{n-1}\right\|^{2}+\left\|e^{n}\right\|^{2}+\left\|e^{n+1}\right\|^{2}+\left\|\xi^{n}\right\|^{2}\right)
$$

for $\tau<\tau_{1}, h<h_{1}$.

Thus, we have

$$
\begin{aligned}
\mid \operatorname{Re} & {\left[-\left(\mathcal{A}_{x}^{\alpha} P^{n}, \Delta_{h}^{\alpha} e^{\bar{n}}\right)\right] \mid } \\
& \leq\left\|\mathcal{A}_{x}^{\alpha} P^{n}\right\|\left\|\Delta_{h}^{\alpha} e^{\bar{n}}\right\| \\
& \leq\left\|P^{n}\right\|\left\|\Delta_{h}^{\alpha} e^{\bar{n}}\right\| \\
& \leq \frac{1}{2 v_{1}}\left\|P^{n}\right\|^{2}+\frac{v_{1}}{2}\left\|\Delta_{h}^{\alpha} e^{\bar{n}}\right\|^{2} \\
& \leq \frac{3 C_{1}^{2}}{2 v_{1}}\left(\left\|e^{n-1}\right\|^{2}+\left\|e^{n}\right\|^{2}+\left\|e^{n+1}\right\|^{2}+\left\|\xi^{n}\right\|^{2}\right)+\frac{v_{1}}{2}\left\|\Delta_{h}^{\alpha} e^{\bar{n}}\right\|^{2} \\
& \leq \frac{9 C_{1}^{2}}{2 v_{1}}\left(\left\|e^{n-1}\right\|_{A}^{2}+\left\|e^{n}\right\|_{A}^{2}+\left\|e^{n+1}\right\|_{A}^{2}+\left\|\xi^{n}\right\|_{A}^{2}\right)+\frac{v_{1}}{2}\left\|\Delta_{h}^{\alpha} e^{\bar{n}}\right\|^{2}
\end{aligned}
$$

and

$$
\left|\operatorname{Re}\left(r^{n}, \Delta_{h}^{\alpha} e^{\bar{n}}\right)\right| \leq\left\|r^{n}\right\|\left\|\Delta_{h}^{\alpha} e^{\bar{n}}\right\| \leq \frac{1}{2 v_{1}}\left\|r^{n}\right\|^{2}+\frac{v_{1}}{2}\left\|\Delta_{h}^{\alpha} e^{\bar{n}}\right\|^{2} .
$$

Substituting (59) and (60) into (57), one has

$$
\begin{aligned}
& \frac{\left\|\Lambda^{\alpha} e^{n+1}\right\|_{A}^{2}-\left\|\Lambda^{\alpha} e^{n-1}\right\|_{A}^{2}}{4 \tau} \\
& \quad \leq \frac{9 C_{1}^{2}}{2 v_{1}}\left(\left\|e^{n-1}\right\|_{A}^{2}+\left\|e^{n}\right\|_{A}^{2}+\left\|e^{n+1}\right\|_{A}^{2}+\left\|\xi^{n}\right\|_{A}^{2}\right)+\left|\gamma_{1}\right|\left\|\Lambda^{\alpha} e^{\bar{n}}\right\|_{A}^{2}+\frac{1}{2 v_{1}}\left\|r^{n}\right\|^{2}
\end{aligned}
$$




$$
\begin{aligned}
\leq & \frac{9 C_{1}^{2}}{2 v_{1}}\left(\left\|e^{n-1}\right\|_{A}^{2}+\left\|e^{n}\right\|_{A}^{2}+\left\|e^{n+1}\right\|_{A}^{2}+\left\|\xi^{n}\right\|_{A}^{2}\right) \\
& +\frac{\left|\gamma_{1}\right|}{2}\left(\left\|\Lambda^{\alpha} e^{n+1}\right\|_{A}^{2}+\left\|\Lambda^{\alpha} e^{n-1}\right\|_{A}^{2}\right)+\frac{1}{2 v_{1}}\left\|r^{n}\right\|^{2} .
\end{aligned}
$$

Thus,

$$
\begin{aligned}
\left\|\Lambda^{\alpha} e^{n+1}\right\|_{A}^{2}-\left\|\Lambda^{\alpha} e^{n-1}\right\|_{A}^{2} & \\
\leq & \frac{18 C_{1}^{2} \tau}{v_{1}}\left(\left\|e^{n-1}\right\|_{A}^{2}+\left\|e^{n}\right\|_{A}^{2}+\left\|e^{n+1}\right\|_{A}^{2}+\left\|\xi^{n}\right\|_{A}^{2}\right) \\
& +2\left|\gamma_{1}\right| \tau\left(\left\|\Lambda^{\alpha} e^{n-1}\right\|_{A}^{2}+\left\|\Lambda^{\alpha} e^{n+1}\right\|_{A}^{2}\right)+\frac{2 \tau}{v_{1}}\left\|r^{n}\right\|^{2}
\end{aligned}
$$

when $\tau<\tau_{1}, h<h_{1}$.

Similarly, computing the discrete inner product of (43) with $\Delta_{h} \xi^{\bar{n}}$ and analyzing the resulting equation, one can obtain

$$
\begin{aligned}
\left\|\Lambda^{\alpha} \xi^{n+1}\right\|_{A}^{2}-\left\|\Lambda^{\alpha} \xi^{n-1}\right\|_{A}^{2} & \\
\leq & \frac{18\left(C_{1}^{\prime}\right)^{2} \tau}{v_{2}}\left(\left\|\xi^{n-1}\right\|_{A}^{2}+\left\|\xi^{n}\right\|_{A}^{2}+\left\|\xi^{n+1}\right\|_{A}^{2}+\left\|e^{n}\right\|_{A}^{2}\right) \\
& +2\left|\gamma_{2}\right| \tau\left(\left\|\Lambda^{\alpha} \xi^{n-1}\right\|_{A}^{2}+\left\|\Lambda^{\alpha} \xi^{n+1}\right\|_{A}^{2}\right)+\frac{2 \tau}{v_{2}}\left\|s^{n}\right\|^{2}
\end{aligned}
$$

when $\tau<\tau_{1}, h<h_{1}$.

Adding inequalities (55), (56), (62), and (63), one obtains

$$
\begin{aligned}
& \left\|\Lambda^{\alpha} e^{n+1}\right\|_{A}^{2}+\left\|\Lambda^{\alpha} \xi^{n+1}\right\|_{A}^{2}+\left\|e^{n+1}\right\|_{A}^{2}+\left\|\xi^{n+1}\right\|_{A}^{2} \\
& \quad-\left\|\Lambda^{\alpha} e^{n-1}\right\|_{A}^{2}-\left\|\Lambda^{\alpha} \xi^{n-1}\right\|_{A}^{2}-\left\|e^{n-1}\right\|_{A}^{2}-\left\|\xi^{n-1}\right\|_{A}^{2} \\
& \leq C_{3} \tau\left(\left\|e^{n-1}\right\|_{A}^{2}+2\left\|e^{n}\right\|_{A}^{2}+\left\|e^{n+1}\right\|_{A}^{2}+\left\|\Lambda^{\alpha} e^{n-1}\right\|_{A}^{2}+2\left\|\Lambda^{\alpha} e^{n}\right\|_{A}^{2}+\left\|\Lambda^{\alpha} e^{n+1}\right\|_{A}^{2}\right) \\
& \quad+C_{3} \tau\left(\left\|\xi^{n-1}\right\|_{A}^{2}+2\left\|\xi^{n}\right\|_{A}^{2}+\left\|\xi^{n+1}\right\|_{A}^{2}+\left\|\Lambda^{\alpha} \xi^{n-1}\right\|_{A}^{2}+2\left\|\Lambda^{\alpha} \xi^{n}\right\|_{A}^{2}+\left\|\Lambda^{\alpha} \xi^{n+1}\right\|_{A}^{2}\right) \\
& \quad+\left(2+\frac{2}{v_{1}}\right) \tau\left\|r^{n}\right\|^{2}+\left(2+\frac{2}{v_{2}}\right) \tau\left\|s^{n}\right\|^{2},
\end{aligned}
$$

where $C_{3}=\max \left\{\frac{18 C_{1}^{2}}{v_{1}}, \frac{18\left(C_{1}^{\prime}\right)^{2}}{v_{2}}, 4 C_{2}, 4 C_{2}^{\prime}, 2\left|\gamma_{1}\right|, 2\left|\gamma_{2}\right|\right\}$.

Let $E^{n}=\left\|\Lambda^{\alpha} e^{n-1}\right\|_{A}^{2}+\left\|e^{n-1}\right\|_{A}^{2}+\left\|\Lambda^{\alpha} e^{n}\right\|_{A}^{2}+\left\|e^{n}\right\|_{A}^{2}+\left\|\Lambda^{\alpha} \xi^{n-1}\right\|_{A}^{2}+\left\|\xi^{n-1}\right\|_{A}^{2}+\left\|\Lambda^{\alpha} \xi^{n}\right\|_{A}^{2}+$ $\left\|\xi^{n}\right\|_{A}^{2}$, then one has

$$
E^{n+1}-E^{n} \leq C_{3} \tau\left(E^{n+1}+E^{n}\right)+\left(2+\frac{2}{v_{1}}\right) \tau\left\|r^{n}\right\|^{2}+\left(2+\frac{2}{v_{2}}\right) \tau\left\|s^{n}\right\|^{2},
$$

which is equivalent to

$$
\left(1-C_{3} \tau\right)\left(E^{n+1}-E^{n}\right) \leq 2 C_{3} \tau E^{n}+\left(2+\frac{2}{v_{1}}\right) \tau\left\|r^{n}\right\|^{2}+\left(2+\frac{2}{v_{2}}\right) \tau\left\|s^{n}\right\|^{2}
$$


When $\tau<\tau_{2}=\frac{1}{2 C_{3}}$, then $1-C_{3} \tau>\frac{1}{2}$, (47) gives

$$
E^{n+1}-E^{n} \leq 4 C_{3} \tau E^{n}+\left(4+\frac{4}{v_{1}}\right) \tau\left\|r^{n}\right\|^{2}+\left(4+\frac{4}{v_{2}}\right) \tau\left\|s^{n}\right\|^{2} .
$$

Replacing $n$ by $k$ in (67) and summing over $k$ from 1 to $n$ yields

$$
E^{n+1}-E^{1} \leq 4 C_{3} \tau \sum_{k=1}^{n} E^{k}+\left(4+\frac{4}{v_{1}}\right) \tau \sum_{k=1}^{n}\left\|r^{k}\right\|^{2}+\left(4+\frac{4}{v_{2}}\right) \tau \sum_{k=1}^{n}\left\|s^{k}\right\|^{2} .
$$

From Lemma 10, one gets

$$
\begin{aligned}
& \tau \sum_{k=1}^{n}\left\|r^{k}\right\|^{2} \leq \tau n C_{R}^{2}\left(\tau^{2}+h^{4}\right)^{2} \leq C_{R}^{2} T\left(\tau^{2}+h^{4}\right)^{2}, \\
& \tau \sum_{k=1}^{n}\left\|s^{k}\right\|^{2} \leq \tau n C_{R}^{2}\left(\tau^{2}+h^{4}\right)^{2} \leq C_{R}^{2} T\left(\tau^{2}+h^{4}\right)^{2} .
\end{aligned}
$$

Lemma 11 yields

$$
\begin{aligned}
\left\|e^{1}\right\|_{A}^{2} & \leq\left\|e^{1}\right\|^{2}=\left(e^{1}, e^{1}\right) \leq h \sum_{j=1}^{M-1}\left|e_{j}^{1}\right|\left|e_{j}^{1}\right| \\
& \leq(b-a) \max _{1 \leq j \leq M-1}\left|e_{j}^{1}\right|^{2} \\
& \leq C_{e}^{2}(b-a)\left(\tau^{2}+\tau h^{2}\right)^{2} \\
& \leq \frac{25}{16} C_{e}^{2}(b-a)\left(\tau^{2}+h^{4}\right)^{2},
\end{aligned}
$$

where $\tau h^{2} \leq \frac{\tau^{2}}{4}+h^{4}$ is used.

And

$$
\begin{aligned}
\left\|\Lambda^{\alpha} e^{1}\right\|_{A}^{2} & \leq\left\|\Lambda^{\alpha} e^{1}\right\|^{2}=\left(\Delta_{h}^{\alpha} e^{1}, e^{1}\right) \leq h \sum_{j=1}^{M-1}\left|\Delta_{h}^{\alpha} e_{j}^{1}\right|\left|e_{j}^{1}\right| \\
& \leq(b-a) \max _{1 \leq j \leq M-1}\left|\Delta_{h}^{\alpha} e_{j}^{1}\right| \max _{1 \leq j \leq M-1}\left|e_{j}^{1}\right| \\
& \leq C_{e}^{2}(b-a)\left(\tau^{2}+\tau h^{2+\alpha}\right)\left(\tau^{2}+\tau h^{2}\right) \\
& \leq \frac{25}{16} C_{e}^{2}(b-a)\left(\tau^{2}+h^{4}\right)^{2}
\end{aligned}
$$

for $h \leq 1$. Thus,

$$
\left\|e^{1}\right\|_{A} \leq \frac{5}{4} C_{e} \sqrt{b-a}\left(\tau^{2}+h^{4}\right), \quad\left\|\Lambda^{\alpha} e^{1}\right\|_{A} \leq \frac{5}{4} C_{e} \sqrt{b-a}\left(\tau^{2}+h^{4}\right)
$$

Similarly,

$$
\left\|\xi^{1}\right\|_{A} \leq \frac{5}{4} C_{e} \sqrt{b-a}\left(\tau^{2}+h^{4}\right), \quad\left\|\Lambda^{\alpha} \xi^{1}\right\|_{A} \leq \frac{5}{4} C_{e} \sqrt{b-a}\left(\tau^{2}+h^{4}\right) .
$$


In addition, $\left\|e^{0}\right\|_{A}=0,\left\|\Lambda^{\alpha} e^{0}\right\|_{A}=0,\left\|\xi^{0}\right\|_{A}=0,\left\|\Lambda^{\alpha} \xi^{0}\right\|_{A}=0$. Thus,

$$
\begin{aligned}
E^{1} & =\left\|\Lambda^{\alpha} e^{1}\right\|_{A}^{2}+\left\|e^{1}\right\|_{A}^{2}+\left\|\Lambda^{\alpha} e^{0}\right\|_{A}^{2}+\left\|e^{0}\right\|_{A}^{2}+\left\|\Lambda^{\alpha} \xi^{1}\right\|_{A}^{2}+\left\|\xi^{1}\right\|_{A}^{2}+\left\|\Lambda^{\alpha} \xi^{0}\right\|_{A}^{2}+\left\|\xi^{0}\right\|_{A}^{2} \\
& \leq\left\|\Lambda^{\alpha} e^{1}\right\|_{A}^{2}+\left\|e^{1}\right\|_{A}^{2}+\left\|\Lambda^{\alpha} \xi^{1}\right\|_{A}^{2}+\left\|\xi^{1}\right\|_{A}^{2} \\
& \leq 25 C_{e}^{2}(b-a)\left(\tau^{2}+h^{4}\right)^{2} .
\end{aligned}
$$

Substituting (69) and (72) into (68) gives

$$
E^{n+1} \leq 4 C_{3} \tau \sum_{k=1}^{n} E^{k}+C_{4}\left(\tau^{2}+h^{4}\right)^{2}
$$

where $C_{4}=25 C_{e}^{2}(b-a)+\left(8+\frac{4}{v_{2}}+\frac{4}{v_{2}}\right) C_{R}^{2} T$.

By using Lemma 9 , one has

$$
E^{n+1} \leq C_{4}\left(\tau^{2}+h^{4}\right)^{2} e^{4 C_{3} \tau n} \leq C_{4} e^{4 C_{3} T}\left(\tau^{2}+h^{4}\right)^{2} .
$$

From Lemma 5, Lemma 6, and Lemma 7, one gets

$$
\begin{aligned}
\frac{1}{3}\left(\left\|e^{n+1}\right\|^{2}+\left(\frac{2}{\pi}\right)^{\alpha}\left|e^{n+1}\right|_{H^{\frac{\alpha}{2}}}^{2}\right) & \leq \frac{1}{3}\left(\left\|e^{n+1}\right\|^{2}+\left\|\Lambda^{\alpha} e^{n+1}\right\|^{2}\right) \\
& \leq\left\|e^{n+1}\right\|_{A}^{2}+\left\|\Lambda^{\alpha} e^{n+1}\right\|_{A}^{2} \leq C_{4} e^{4 C_{3} T}\left(\tau^{2}+h^{4}\right)^{2} .
\end{aligned}
$$

Thus, one obtains that

$$
\left\|e^{n+1}\right\|_{H^{\frac{\alpha}{2}}}^{2} \leq 3 C_{4} e^{4 C_{3} T}\left(\frac{\pi}{2}\right)^{\alpha}\left(\tau^{2}+h^{4}\right)^{2} .
$$

Therefore,

$$
\left\|e^{n+1}\right\|_{H^{\frac{\alpha}{2}}} \leq C_{5}\left(\tau^{2}+h^{4}\right)
$$

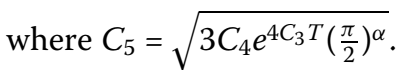

By Lemma 4, we obtain

$$
\left\|e^{n+1}\right\|_{\infty} \leq C_{\alpha}\left\|e^{n+1}\right\|_{H^{\frac{\alpha}{2}}} \leq C_{\alpha} C_{5}\left(\tau^{2}+h^{4}\right) .
$$

Similarly, we also have

$$
\left\|\xi^{n+1}\right\|_{\infty} \leq C_{\alpha}\left\|\xi^{n+1}\right\|_{H^{\frac{\alpha}{2}}} \leq C_{\alpha} C_{5}\left(\tau^{2}+h^{4}\right) .
$$

Now we take $C_{0}=\max \left\{C_{\alpha} C_{5}, \frac{5}{4} C_{e}\right\}$. Once $C_{0}$ is fixed, the condition for $\tau_{1}, h_{1}$, i.e., $\tau_{1}^{2}+h_{1}^{4}<$ $1 / C_{0}$, can be used to determine $\tau_{1}, h_{1}$.

Thus, let $\tau_{0}=\min \left\{\tau_{1}, \tau_{2}\right\}$ and $h_{0}=\min \left\{h_{1}, 1\right\}$, then (46) is valid for $n+1$. The induction is closed. This completes the proof. 
Theorem 2 Suppose that the solution of problem (1)-(3) is smooth enough, the solution $U^{n}$ of difference schemes (22)-(25) and (30)-(31) is bounded in the $L^{\infty}$-norm for $\tau<\tau_{0}$ and $h<h_{0}$, i.e.,

$$
\left\|U^{n}\right\|_{\infty} \leq C_{*}, \quad\left\|V^{n}\right\|_{\infty} \leq C_{*}, \quad 1 \leq n \leq N
$$

where $\tau_{0}, h_{0}$ are the same positive constants in Theorem 1.

Proof From Theorem 1, one has

$$
\begin{aligned}
\left\|U^{n}\right\|_{\infty} & \leq\left\|u^{n}\right\|_{\infty}+\left\|e^{n}\right\|_{\infty}, \\
& \leq C_{m}+C_{0}\left(\tau^{2}+h^{4}\right), \quad 1 \leq n \leq N,
\end{aligned}
$$

and

$$
\begin{aligned}
\left\|V^{n}\right\|_{\infty} & \leq\left\|v^{n}\right\|_{\infty}+\left\|\xi^{n}\right\|_{\infty}, \\
& \leq C_{m}+C_{0}\left(\tau^{2}+h^{4}\right), \quad 1 \leq n \leq N,
\end{aligned}
$$

for $\tau<\tau_{0}, h<h_{0}$.

Since $C_{0}\left(\tau^{2}+h^{4}\right)<1$ for $\tau<\tau_{0}, h<h_{0}$ (see the proof in the above theorem), one has

$$
\left\|U^{n}\right\|_{\infty} \leq C_{*}, \quad\left\|V^{n}\right\|_{\infty} \leq C_{*}, \quad 2 \leq n \leq N
$$

where $C_{*}=C_{m}+1$. This completes the proof.

\subsection{Existence and uniqueness}

Theorem 3 Suppose that the solution of problem (1)-(3) is smooth enough. Then difference scheme (22)-(25) is uniquely solvable for

$$
\tau<\min \left\{\tau_{0}, \frac{1}{\left|\gamma_{1}\right|+\left(\sqrt{\kappa_{1}^{2}+\zeta_{1}^{2}}+\sqrt{\delta_{1}^{2}+\beta_{1}^{2}}\right) C_{*}^{2}}, \frac{1}{\left|\gamma_{2}\right|+\left(\sqrt{\kappa_{2}^{2}+\zeta_{2}^{2}}+\sqrt{\delta_{2}^{2}+\beta_{2}^{2}}\right) C_{*}^{2}}\right\}
$$

and $h<h_{0}$, where $\tau_{0}, h_{0}$ are the same positive constants in Theorem 1.

Proof To prove the theorem, we proceed by the mathematical induction. Obviously, $U^{1}$ and $V^{1}$ can be uniquely determined by (30) and (31). Suppose $U^{1}, U^{2}, \ldots, U^{n}$, $V^{1}, V^{2}, \ldots, V^{n}(1 \leq n \leq N-1)$ are obtained uniquely, we now show that $U^{n+1}$ and $V^{n+1}$ are uniquely determined by (22)-(23).

Assume that $U^{n+1,1}, U^{n+1,2}$ are two solutions of (22), and let $W^{n+1}=U^{n+1,1}-U^{n+1,2}$, then it is easy to verify that $W^{n+1}$ satisfies the following equation:

$$
\begin{aligned}
& \frac{1}{2 \tau} \mathcal{A}_{x}^{\alpha} W_{j}^{n+1}+\frac{1}{2}\left(v_{1}+i \eta_{1}\right) \Delta_{h}^{\alpha} W_{j}^{n+1}+\frac{1}{2} \mathcal{A}_{x}^{\alpha}\left(\left(\left(\kappa_{1}+i \zeta_{1}\right)\left|U_{j}^{n}\right|^{2}+\left(\delta_{1}+i \beta_{1}\right)\left|V_{j}^{n}\right|^{2}\right) W_{j}^{n+1}\right) \\
& \quad-\frac{1}{2} \gamma_{1} \mathcal{A}_{x}^{\alpha} W_{j}^{n+1}=0, \quad 0<j<M
\end{aligned}
$$


Computing the inner product of (84) with $W^{n+1}$ and taking the real part of the resulting equation, we have

$$
\begin{aligned}
& \left(\frac{1}{2 \tau}-\frac{\gamma_{1}}{2}\right)\left\|W^{n+1}\right\|_{A}^{2}+\frac{v_{1}}{2}\left\|\Lambda^{\alpha} W^{n+1}\right\|^{2} \\
& \quad+\frac{1}{2} \operatorname{Re}\left(\mathcal{A}_{x}^{\alpha}\left(\left(\left(\kappa_{1}+i \zeta_{1}\right)\left|U^{n}\right|^{2}+\left(\delta_{1}+i \beta_{1}\right)\left|V^{n}\right|^{2}\right) W^{n+1}\right), W^{n+1}\right)=0,
\end{aligned}
$$

where Lemma 7 is used.

Thus,

$$
\begin{aligned}
& \left(\frac{1}{\tau}-\gamma_{1}\right)\left\|W^{n+1}\right\|_{A}^{2}+v_{1}\left\|\Lambda^{\alpha} W^{n+1}\right\|^{2} \\
& \quad=-\operatorname{Re}\left(\mathcal{A}_{x}^{\alpha}\left(\left(\left(\kappa_{1}+i \zeta_{1}\right)\left|U^{n}\right|^{2}+\left(\delta_{1}+i \beta_{1}\right)\left|V^{n}\right|^{2}\right) W^{n+1}\right), W^{n+1}\right) \\
& \quad=-\operatorname{Re}\left(\left(\left(\kappa_{1}+i \zeta_{1}\right)\left|U^{n}\right|^{2}+\left(\delta_{1}+i \beta_{1}\right)\left|V^{n}\right|^{2}\right) W^{n+1}, \mathcal{A}_{x}^{\alpha} W^{n+1}\right) \\
& \quad \leq\left(\sqrt{\kappa_{1}^{2}+\zeta_{1}^{2}}+\sqrt{\delta_{1}^{2}+\beta_{1}^{2}}\right) C_{*}^{2}\left\|W^{n+1}\right\|_{A}^{2}
\end{aligned}
$$

when $\tau<\tau_{0}, h<h_{0}$, and here Theorem 2 is used.

If $\tau<\tau_{0}, h<h_{0}$, then

$$
\left(\frac{1}{\tau}-\gamma_{1}-\left(\sqrt{\kappa_{1}^{2}+\zeta_{1}^{2}}+\sqrt{\delta_{1}^{2}+\beta_{1}^{2}}\right) C_{*}^{2}\right)\left\|W^{n+1}\right\|_{A}^{2}+v_{1}\left\|\Lambda^{\alpha} W^{n+1}\right\|^{2} \leq 0,
$$

where Theorem 2 is used.

If $\tau<\min \left\{\tau_{0}, \frac{1}{\left|\gamma_{1}\right|+\left(\sqrt{\kappa_{1}^{2}+\zeta_{1}^{2}}+\sqrt{\delta_{1}^{2}+\beta_{1}^{2}}\right) C_{*}^{2}}\right\}$, then $\frac{1}{\tau}-\gamma_{1}-\left(\sqrt{\kappa_{1}^{2}+\zeta_{1}^{2}}+\sqrt{\delta_{1}^{2}+\beta_{1}^{2}}\right) C_{*}^{2}>0$. Since $v_{1}>0$, (87) implies

$$
\left\|W^{n+1}\right\|_{A}=\left\|\Lambda^{\alpha} W^{n+1}\right\|=0,
$$

which further implies

$$
\left\|W^{n+1}\right\|=\left\|\Lambda^{\alpha} W^{n+1}\right\|=0
$$

where Lemma 6 is used.

Therefore, (84) has only a trivial solution. This proves the uniqueness of the numerical solution $U^{n+1}$. The proof of the uniqueness for $V^{n+1}$ is similar. This completes the proof of the theorem.

\subsection{Stability}

Next we will show that the numerical solutions of the proposed scheme are stable in the sense that when initial conditions (3) and the right-hand sides of equations (1)-(2) have small perturbations, the perturbation for the numerical solutions remains small. Consider the following two problems.

\section{Problem 1}

$$
\begin{aligned}
u_{t} & +\left(v_{1}+i \eta_{1}\right)(-\Delta)^{\frac{\alpha}{2}} u+\left(\left(\kappa_{1}+i \zeta_{1}\right)|u|^{2}+\left(\delta_{1}+i \beta_{1}\right)|v|^{2}\right) u-\gamma_{1} u \\
& =f_{1}(x, t), \quad x \in[a, b], 0<t<T,
\end{aligned}
$$




$$
\begin{aligned}
v_{t} & +\left(v_{2}+i \eta_{2}\right)(-\Delta)^{\frac{\alpha}{2}} v+\left(\left(\kappa_{2}+i \zeta_{2}\right)|u|^{2}+\left(\delta_{2}+i \beta_{2}\right)|v|^{2}\right) v-\gamma_{2} v \\
& =g_{1}(x, t), \quad x \in[a, b], 0<t<T,
\end{aligned}
$$

with the initial condition

$$
u(x, 0)=u_{1}(x), \quad v(x, 0)=v_{1}(x), \quad x \in[a, b], 0<t<T,
$$

and the homogeneous Dirichlet boundary conditions

$$
u_{1}(x, t)=0, \quad v_{1}(x, t)=0, \quad x \in \mathbb{R} /(a, b), t \in[0, T] .
$$

\section{Problem 2}

$$
\begin{aligned}
u_{t} & +\left(v_{1}+i \eta_{1}\right)(-\Delta)^{\frac{\alpha}{2}} u+\left(\left(\kappa_{1}+i \zeta_{1}\right)|u|^{2}+\left(\delta_{1}+i \beta_{1}\right)|v|^{2}\right) u-\gamma_{1} u \\
& =f_{2}(x, t), \quad x \in[a, b], 0<t<T, \\
v_{t} & +\left(v_{2}+i \eta_{2}\right)(-\Delta)^{\frac{\alpha}{2}} v+\left(\left(\kappa_{2}+i \zeta_{2}\right)|u|^{2}+\left(\delta_{2}+i \beta_{2}\right)|v|^{2}\right) v-\gamma_{2} v \\
& =g_{2}(x, t), \quad x \in[a, b], 0<t<T,
\end{aligned}
$$

with the initial condition

$$
u(x, 0)=u_{2}(x), \quad v(x, 0)=v_{2}(x), \quad x \in[a, b], 0<t<T,
$$

and the homogeneous Dirichlet boundary conditions

$$
u_{2}(x, t)=0, \quad v_{2}(x, t)=0, \quad x \in \mathbb{R} /(a, b), t \in[0, T] .
$$

The numerical solutions $U_{j}^{n}, V_{j}^{n}$ for the first problem are given as follows:

$$
\begin{aligned}
& \mathcal{A}_{x}^{\alpha} \delta_{t} U_{j}^{n}+\left(v_{1}+i \eta_{1}\right) \Delta_{h}^{\alpha} U_{j}^{\bar{n}}+\mathcal{A}_{x}^{\alpha}\left[\left(\left(\kappa_{1}+i \zeta_{1}\right)\left|U_{j}^{n}\right|^{2}+\left(\delta_{1}+i \beta_{1}\right)\left|V_{j}^{n}\right|^{2}\right) U_{j}^{\bar{n}}\right]-\gamma_{1} \mathcal{A}_{x}^{\alpha} U_{j}^{\bar{n}} \\
& =f_{1}\left(x_{j}, t^{n}\right), \quad 0<j<M, 1<n<N, \\
& \mathcal{A}_{x}^{\alpha} \delta_{t} V_{j}^{n}+\left(v_{2}+i \eta_{2}\right) \Delta_{h}^{\alpha} V_{j}^{\bar{n}}+\mathcal{A}_{x}^{\alpha}\left[\left(\left(\kappa_{2}+i \zeta_{2}\right)\left|U_{j}^{n}\right|^{2}+\left(\delta_{2}+i \beta_{2}\right)\left|V_{j}^{n}\right|^{2}\right) V_{j}^{\bar{n}}\right]-\gamma_{2} \mathcal{A}_{x}^{\alpha} V_{j}^{\bar{n}} \\
& =g_{1}\left(x_{j}, t^{n}\right), \quad 0<j<M, 1<n<N, \\
& U_{j}^{1}=U_{j}^{0}-\tau\left(\left(v_{1}+i \eta_{1}\right) \Delta_{h}^{\alpha} U_{j}^{0}\right. \\
& \left.+\left(\left(\kappa_{1}+i \zeta_{1}\right)\left|U_{j}^{0}\right|^{2}+\left(\delta_{1}+i \beta_{1}\right)\left|V_{j}^{0}\right|^{2}\right) U_{j}^{0}-\gamma_{1} U_{j}^{0}-f_{1}\left(x_{j}, t^{0}\right)\right), \quad 0<j<M, \\
& V_{j}^{1}=V_{j}^{0}-\tau\left(\left(v_{2}+i \eta_{2}\right) \Delta_{h}^{\alpha} V_{j}^{0}\right. \\
& \left.+\left(\left(\kappa_{2}+i \zeta_{2}\right)\left|U_{j}^{0}\right|^{2}+\left(\delta_{2}+i \beta_{2}\right)\left|V_{j}^{0}\right|^{2}\right) V_{j}^{0}-\gamma_{2} V_{j}^{0}-g_{1}\left(x_{j}, t^{0}\right)\right), \quad 0<j<M, \\
& U_{j}^{0}=u_{1}\left(x_{j}\right), \quad V_{j}^{0}=v_{1}\left(x_{j}\right), \quad 0<j<M, \\
& U_{0}^{n}=U_{M}^{n}=0, \quad V_{0}^{n}=V_{M}^{n}=0, \quad 0 \leq n \leq N .
\end{aligned}
$$


The numerical solutions $\mathcal{U}_{j}^{n}, \mathcal{V}_{j}^{n}$ for the second problem are given as follows:

$$
\begin{aligned}
& \mathcal{A}_{x}^{\alpha} \delta_{t} \mathcal{U}_{j}^{n}+\left(v_{1}+i \eta_{1}\right) \Delta_{h}^{\alpha} \mathcal{U}_{j}^{\bar{n}}+\mathcal{A}_{x}^{\alpha}\left[\left(\left(\kappa_{1}+i \zeta_{1}\right)\left|\mathcal{U}_{j}^{n}\right|^{2}+\left(\delta_{1}+i \beta_{1}\right)\left|\mathcal{V}_{j}^{n}\right|^{2}\right) \mathcal{U}_{j}^{\bar{n}}\right]-\gamma_{1} \mathcal{A}_{x}^{\alpha} U_{j}^{\bar{n}} \\
& =f_{2}\left(x_{j}, t^{n}\right), \quad 0<j<M, 1<n<N, \\
& \mathcal{A}_{x}^{\alpha} \delta_{t} \mathcal{V}_{j}^{n}+\left(v_{2}+i \eta_{2}\right) \Delta_{h}^{\alpha} \mathcal{V}_{j}^{\bar{n}}+\mathcal{A}_{x}^{\alpha}\left[\left(\left(\kappa_{2}+i \zeta_{2}\right)\left|\mathcal{U}_{j}^{n}\right|^{2}+\left(\delta_{2}+i \beta_{2}\right)\left|\mathcal{V}_{j}^{n}\right|^{2}\right) \mathcal{V}_{j}^{\bar{n}}\right]-\gamma_{2} \mathcal{A}_{x}^{\alpha} \mathcal{V}_{j}^{\bar{n}} \\
& =g_{2}\left(x_{j}, t^{n}\right), \quad 0<j<M, 1<n<N \\
& \mathcal{U}_{j}^{1}=\mathcal{U}_{j}^{0}-\tau\left(\left(v_{1}+i \eta_{1}\right) \Delta_{h}^{\alpha} \mathcal{U}_{j}^{0}\right. \\
& \left.+\left(\left(\kappa_{1}+i \zeta_{1}\right)\left|\mathcal{U}_{j}^{0}\right|^{2}+\left(\delta_{1}+i \beta_{1}\right)\left|\mathcal{V}_{j}^{0}\right|^{2}\right) \mathcal{U}_{j}^{0}-\gamma_{1} \mathcal{U}_{j}^{0}-f_{2}\left(x_{j}, t^{0}\right)\right), \quad 0<j<M, \\
& \mathcal{V}_{j}^{1}=\mathcal{V}_{j}^{0}-\tau\left(\left(v_{2}+i \eta_{2}\right) \Delta_{h}^{\alpha} \mathcal{V}_{j}^{0}\right. \\
& \left.+\left(\left(\kappa_{2}+i \zeta_{2}\right)\left|\mathcal{U}_{j}^{0}\right|^{2}+\left(\delta_{2}+i \beta_{2}\right)\left|\mathcal{V}_{j}^{0}\right|^{2}\right) \mathcal{V}_{j}^{0}-\gamma_{2} \mathcal{V}_{j}^{0}-g_{2}\left(x_{j}, t^{0}\right)\right), \quad 0<j<M \\
& \mathcal{U}_{j}^{0}=u_{2}\left(x_{j}\right), \quad \mathcal{V}_{j}^{0}=v_{2}\left(x_{j}\right), \quad 0<j<M, \\
& \mathcal{U}_{0}^{n}=\mathcal{U}_{M}^{n}=0, \quad \mathcal{V}_{0}^{n}=\mathcal{V}_{M}^{n}=0, \quad 0 \leq n \leq N
\end{aligned}
$$

Now denote

$$
\begin{aligned}
& \quad \hat{U}_{j}^{n}=U_{j}^{n}-\mathcal{U}_{j}^{n}, \quad \hat{V}_{j}^{n}=V_{j}^{n}-\mathcal{V}_{j}^{n}, \\
& \hat{f}_{j}^{n}=f_{1}\left(x_{j}, t^{n}\right)-f_{2}\left(x_{j}, t^{n}\right), \quad \hat{g}_{j}^{n}=g_{1}\left(x_{j}, t^{n}\right)-g_{2}\left(x_{j}, t^{n}\right), \\
& \hat{u}_{j}=u_{1}\left(x_{j}\right)-u_{2}\left(x_{j}\right), \quad \hat{v}_{j}=v_{1}\left(x_{j}\right)-v_{2}\left(x_{j}\right), \\
& \text { where } j=1,2, \ldots, M, n=1,2, \ldots, N .
\end{aligned}
$$

Subtracting (98) from (104) and subtracting (99) from (105) yield that

$$
\begin{aligned}
& \hat{f}_{j}^{n}=\mathcal{A}_{x}^{\alpha} \delta_{t} \hat{U}_{j}^{n}+\left(v_{1}+i \eta_{1}\right) \Delta_{h}^{\alpha} \hat{U}_{j}^{\bar{n}}+\mathcal{A}_{x}^{\alpha} R_{j}^{n}-\gamma_{1} \mathcal{A}_{x}^{\alpha} \hat{U}_{j}^{\bar{n}}, \\
& \hat{g}_{j}^{n}=\mathcal{A}_{x}^{\alpha} \delta_{t} \hat{V}_{j}^{n}+\left(v_{2}+i \eta_{2}\right) \Delta_{h}^{\alpha} \hat{V}_{j}^{\bar{n}}+\mathcal{A}_{x}^{\alpha} S_{j}^{n}-\gamma_{2} \mathcal{A}_{x}^{\alpha} \hat{V}_{j}^{\bar{n}}
\end{aligned}
$$

where

$$
\begin{aligned}
& R_{j}^{n}=\left(\left(\kappa_{1}+i \zeta_{1}\right)\left|U_{j}^{n}\right|^{2}+\left(\delta_{1}+i \beta_{1}\right)\left|V_{j}^{n}\right|^{2}\right) U_{j}^{\bar{n}}-\left(\left(\kappa_{1}+i \zeta_{1}\right)\left|\mathcal{U}_{j}^{n}\right|^{2}+\left(\delta_{1}+i \beta_{1}\right)\left|\mathcal{V}_{j}^{n}\right|^{2}\right) \mathcal{U}_{j}^{\bar{n}}, \\
& S_{j}^{n}=\left(\left(\kappa_{2}+i \zeta_{2}\right)\left|U_{j}^{n}\right|^{2}+\left(\delta_{2}+i \beta_{2}\right)\left|V_{j}^{n}\right|^{2}\right) V_{j}^{\bar{n}}-\left(\left(\kappa_{2}+i \zeta_{2}\right)\left|\mathcal{U}_{j}^{n}\right|^{2}+\left(\delta_{2}+i \beta_{2}\right)\left|\mathcal{V}_{j}^{n}\right|^{2}\right) \mathcal{V}_{j}^{\bar{n}}
\end{aligned}
$$

for $1 \leq j \leq M-1$ and $1 \leq n \leq N-1$.

Subtracting (100) from (106) and subtracting (101) from (107) yield that

$$
\begin{aligned}
& \hat{U}_{j}^{1}=\hat{U}_{j}^{0}-\tau\left(\left(v_{1}+i \eta_{1}\right) \Delta_{h}^{\alpha} \hat{U}_{j}^{0}+R_{j}^{n}-\gamma_{1} \hat{U}_{j}^{0}-\hat{f}_{j}^{0}\right), \\
& \hat{V}_{j}^{1}=\hat{V}_{j}^{0}-\tau\left(\left(v_{2}+i \eta_{2}\right) \Delta_{h}^{\alpha} \hat{V}_{j}^{0}+S_{j}^{n}-\gamma_{2} \hat{V}_{j}^{0}-\hat{g}_{j}^{0}\right),
\end{aligned}
$$

where

$$
\begin{aligned}
& R_{j}^{1}=\left(\left(\kappa_{1}+i \zeta_{1}\right)\left|U_{j}^{0}\right|^{2}+\left(\delta_{1}+i \beta_{1}\right)\left|V_{j}^{0}\right|^{2}\right) U_{j}^{0}-\left(\left(\kappa_{1}+i \zeta_{1}\right)\left|\mathcal{U}_{j}^{0}\right|^{2}+\left(\delta_{1}+i \beta_{1}\right)\left|\mathcal{V}_{j}^{0}\right|^{2}\right) \mathcal{U}_{j}^{0}, \\
& S_{j}^{1}=\left(\left(\kappa_{2}+i \zeta_{2}\right)\left|U_{j}^{0}\right|^{2}+\left(\delta_{2}+i \beta_{2}\right)\left|V_{j}^{0}\right|^{2}\right) V_{j}^{0}-\left(\left(\kappa_{2}+i \zeta_{2}\right)\left|\mathcal{U}_{j}^{0}\right|^{2}+\left(\delta_{2}+i \beta_{2}\right)\left|\mathcal{V}_{j}^{0}\right|^{2}\right) \mathcal{V}_{j}^{0}
\end{aligned}
$$


Following a simple analysis, one can easily show the following lemma.

Lemma 12 Suppose that the initial conditions and the solutions of the above two problems are sufficiently smooth. Then one has

$$
\begin{aligned}
& \left|\hat{U}_{j}^{1}\right| \leq C_{p}\left(\left|\hat{u}_{j}\right|+\left|\hat{v}_{j}\right|+\left|\Delta_{h}^{\alpha} \hat{u}_{j}\right|+\tau\left|\hat{f}_{j}^{0}\right|\right), \\
& \left|\Delta_{h}^{\alpha} \hat{U}_{j}^{1}\right| \leq C_{p}\left(\left|\Delta_{h}^{\alpha} \hat{u}_{j}\right|+\left|\Delta_{h}^{\alpha} \hat{v}_{j}\right|+\left|\Delta_{h}^{\alpha} \Delta_{h}^{\alpha} \hat{u}_{j}\right|+\tau\left|\Delta_{h}^{\alpha} \hat{f}_{j}^{0}\right|\right), \\
& \left|\hat{V}_{j}^{1}\right| \leq C_{p}\left(\left|\hat{u}_{j}\right|+\left|\hat{v}_{j}\right|+\left|\Delta_{h}^{\alpha} \hat{v}_{j}\right|+\tau\left|\hat{g}_{j}^{0}\right|\right), \\
& \left|\Delta_{h}^{\alpha} \hat{V}_{j}^{1}\right| \leq C_{p}\left(\left|\Delta_{h}^{\alpha} \hat{u}_{j}\right|+\left|\Delta_{h}^{\alpha} \hat{v}_{j}\right|+\left|\Delta_{h}^{\alpha} \Delta_{h}^{\alpha} \hat{v}_{j}\right|+\tau\left|\Delta_{h}^{\alpha} \hat{g}_{j}^{0}\right|\right), \quad 0 \leq j \leq M,
\end{aligned}
$$

where $C_{p}$ is a positive constant independent of $\tau$ and $h$.

By using Lemma 12, the $L^{\infty}$-norm boundedness of the numerical solutions $U^{n}, V^{n}, \mathcal{U}^{n}$, $\mathcal{V}^{n}$ from Theorem 2, the discrete Gronwall's inequality of Lemma 9, the similar type of mathematical induction, and inner product technics as shown in the proof of Theorem 1 , one can show the following stability results (the detailed proof is omitted).

Theorem 4 Suppose that the solutions of the above two problems are smooth enough, then there exist two small positive constants $\tau^{0}$ and $h^{0}$ such that, when $\tau<\tau^{0}$ and $h<h^{0}$, the numerical solution $\left(U^{n}, V^{n}\right)$ of difference scheme (98)-(103) and the numerical solution $\left(\mathcal{U}^{n}, \mathcal{V}^{n}\right)$ of difference scheme (104)-(109) have the following estimates:

$$
\begin{aligned}
\| U^{n}- & \mathcal{U}^{n} \|_{\infty}^{2} \\
\leq & C^{0}\left(\|\hat{u}\|^{2}+\|\hat{v}\|^{2}+\left\|\Delta_{h}^{\alpha} \hat{u}\right\|^{2}+\left\|\Delta_{h}^{\alpha} \hat{v}\right\|^{2}+\left\|\Delta_{h}^{\alpha} \Delta_{h}^{\alpha} \hat{u}\right\|^{2}+\left\|\Delta_{h}^{\alpha} \Delta_{h}^{\alpha} \hat{v}\right\|^{2}\right) \\
& +C^{0}\left(\tau^{2}\left\|\hat{f}^{0}\right\|^{2}+\tau^{2}\left\|\hat{g}^{0}\right\|^{2}+\tau^{2}\left\|\Delta_{h}^{\alpha} \hat{f}^{0}\right\|^{2}+\tau^{2}\left\|\Delta_{h}^{\alpha} \hat{g}^{0}\right\|^{2}\right. \\
& \left.+\tau \sum_{k=1}^{n-1}\left\|\hat{f}^{k}\right\|^{2}+\tau \sum_{k=1}^{n-1}\left\|\hat{g}^{k}\right\|^{2}\right), \\
\left\|V^{n}-\mathcal{V}^{n}\right\|_{\infty}^{2} & C^{0}\left(\|\hat{u}\|^{2}+\|\hat{v}\|^{2}+\left\|\Delta_{h}^{\alpha} \hat{u}\right\|^{2}+\left\|\Delta_{h}^{\alpha} \hat{v}\right\|_{2}^{2}+\left\|\Delta_{h}^{\alpha} \Delta_{h}^{\alpha} \hat{u}\right\|^{2}+\left\|\Delta_{h}^{\alpha} \Delta_{h}^{\alpha} \hat{v}\right\|^{2}\right) \\
& +C^{0}\left(\tau^{2}\left\|\hat{f}^{0}\right\|^{2}+\tau^{2}\left\|\hat{g}^{0}\right\|^{2}+\tau^{2}\left\|\Delta_{h}^{\alpha} \hat{f}^{0}\right\|^{2}+\tau^{2}\left\|\Delta_{h}^{\alpha} \hat{g}^{0}\right\|^{2}\right. \\
& \left.+\tau \sum_{k=1}^{n-1}\left\|\hat{f}^{k}\right\|^{2}+\tau \sum_{k=1}^{n-1}\left\|\hat{g}^{k}\right\|^{2}\right), \quad 1 \leq n \leq N,
\end{aligned}
$$

where $C^{0}$ is a positive constant independent of $\tau$ and $h$.

From the above theorem, one can see that if the initial perturbations $\hat{u}, \hat{v}$ and the perturbations of the right-hand sides $\hat{f}, \hat{g}$ are small, then the difference of numerical solutions corresponding to these perturbations remains small. Therefore, the method proposed in this paper is unconditionally stable. 
Remark 1 In order to increase the time accuracy, the following Richardson extrapolation for the final step numerical solution is used:

$$
\begin{aligned}
& \widetilde{U}^{N}(\Delta t, h)=\frac{4}{3} U^{N}(\Delta t, h)-\frac{1}{3} U^{N / 2}(2 \Delta t, h), \\
& \widetilde{V}^{N}(\Delta t, h)=\frac{4}{3} V^{N}(\Delta t, h)-\frac{1}{3} V^{N / 2}(2 \Delta t, h),
\end{aligned}
$$

where $U^{N}(\Delta t, h), U^{N / 2}(2 \Delta t, h)$ are numerical solutions at the final step by using spatial meshsizes $h$ and time step $\Delta t, 2 \Delta t$, respectively. We will use this temporal scheme for numerical computation.

\section{Numerical results}

In this section, we present some numerical results of the proposed difference scheme (22)(25) to support our theoretical findings.

Example 1 In order to test the accuracy of the proposed scheme, we consider the following system with source terms:

$$
\begin{aligned}
& u_{t}+(1+i)(-\Delta)^{\frac{\alpha}{2}} u+\left((-1-i)|u|^{2}+(1+i)|v|^{2}\right) u-u=f(x, t), \quad x \in(0,1), 0<t<1, \\
& v_{t}+(1-i)(-\Delta)^{\frac{\alpha}{2}} v+\left((1+i)|u|^{2}+(1-i)|v|^{2}\right) v+v=g(x, t), \quad x \in(0,1), 0<t<1,
\end{aligned}
$$

with the homogeneous boundary conditions

$$
u(0, t)=u(1, t)=0, \quad v(0, t)=v(1, t)=0 .
$$

The initial conditions and the source terms $f(x, t)$ and $g(x, t)$ are determined by the exact solutions

$$
u(x, t)=\exp (-t) x^{4}(1-x)^{4}, \quad v(x, t)=(t+1)^{3} x^{4}(1-x)^{4} .
$$

Table 1 and Table 2 list the errors and the convergence orders for the proposed method with $\alpha=1.2,1.5,1.8,2.0$ in the $L^{\infty}$-norm, respectively. As we can see, these results show that the proposed method is fourth-order convergence both in time and space variables.

In order the illustrate the unconditional stability of our methods, we fix $\tau$ and vary $h$,

\begin{tabular}{|c|c|c|c|c|c|c|c|c|c|}
\hline \multirow[t]{2}{*}{$\tau$} & \multirow[t]{2}{*}{$h$} & \multicolumn{2}{|l|}{$\alpha=1.2$} & \multicolumn{2}{|l|}{$\alpha=1.5$} & \multicolumn{2}{|l|}{$\alpha=1.8$} & \multicolumn{2}{|l|}{$\alpha=2.0$} \\
\hline & & $\left\|u^{n}-\widetilde{U}^{n}\right\|_{\infty}$ & $\overline{\text { Order }}$ & $\left\|u^{n}-\widetilde{U}^{n}\right\|_{\infty}$ & $\overline{\text { Order }}$ & $\left\|u^{n}-\tilde{U}^{n}\right\|_{\infty}$ & $\overline{\text { Order }}$ & $\left\|u^{n}-\widetilde{U}^{n}\right\|_{\infty}$ & Order \\
\hline $1 / 32$ & $1 / 32$ & $1.61 \mathrm{e}-08$ & - & $2.83 \mathrm{e}-08$ & - & $1.05 \mathrm{e}-07$ & - & $1.68 \mathrm{e}-07$ & - \\
\hline $1 / 64$ & $1 / 64$ & $8.78 \mathrm{e}-10$ & 4.20 & $1.81 \mathrm{e}-09$ & 3.97 & $2.66 \mathrm{e}-09$ & 5.31 & $3.15 \mathrm{e}-09$ & 5.74 \\
\hline $1 / 128$ & $1 / 128$ & $5.75 \mathrm{e}-11$ & 3.93 & $1.05 \mathrm{e}-10$ & 4.11 & $1.48 \mathrm{e}-10$ & 4.16 & $2.24 \mathrm{e}-10$ & 3.81 \\
\hline $1 / 256$ & $1 / 256$ & $3.93 e-12$ & 3.87 & $6.14 \mathrm{e}-12$ & 4.09 & $8.99 \mathrm{e}-12$ & 4.05 & $1.30 \mathrm{e}-11$ & 4.11 \\
\hline $1 / 512$ & $1 / 512$ & $2.67 e-13$ & 3.88 & $3.63 e-13$ & 4.08 & $5.40 e-13$ & 4.06 & $8.07 e-13$ & 4.01 \\
\hline
\end{tabular}
results for $\alpha=1.5$ and $\alpha=2$ are plotted in Fig. 1. As one can see, these results clearly show that the time step is not related to the spatial meshsize, and as the spatial meshsize goes to zero, the dominant error comes from the temporal part.

Table $1 L^{\infty}$-norm errors and convergence orders of $\widetilde{U}$ obtained by the fourth-order scheme for Example 1 
Table $2 L^{\infty}$-norm errors and convergence orders of $\tilde{V}$ obtained by the fourth-order scheme for Example 1

\begin{tabular}{|c|c|c|c|c|c|c|c|c|c|}
\hline \multirow[t]{2}{*}{$\tau$} & \multirow[t]{2}{*}{ h } & \multicolumn{2}{|l|}{$\alpha=1.2$} & \multicolumn{2}{|l|}{$\alpha=1.5$} & \multicolumn{2}{|l|}{$\alpha=1.8$} & \multicolumn{2}{|l|}{$\alpha=2.0$} \\
\hline & & $\left\|v^{n}-\tilde{V}^{n}\right\|_{\infty}$ & Order & $\left\|v^{n}-\widetilde{V}^{n}\right\|_{\infty}$ & Order & $\left\|v^{n}-\widetilde{V}^{n}\right\|_{\infty}$ & Order & $\left\|v^{n}-\widetilde{V}^{n}\right\|_{\infty}$ & Order \\
\hline $1 / 32$ & $1 / 32$ & $3.42 \mathrm{e}-07$ & - & $5.19 \mathrm{e}-07$ & - & $1.08 \mathrm{e}-06$ & - & $1.29 \mathrm{e}-06$ & - \\
\hline $1 / 64$ & $1 / 64$ & $1.96 \mathrm{e}-08$ & 4.12 & $3.04 \mathrm{e}-08$ & 4.09 & $4.62 \mathrm{e}-08$ & 4.55 & $5.69 \mathrm{e}-08$ & 4.50 \\
\hline $1 / 128$ & $1 / 128$ & $1.16 \mathrm{e}-09$ & 4.08 & $1.78 e-09$ & 4.09 & $2.79 \mathrm{e}-09$ & 4.05 & $4.10 \mathrm{e}-09$ & 3.80 \\
\hline $1 / 256$ & $1 / 256$ & $7.76 \mathrm{e}-11$ & 3.90 & $1.05 e-10$ & 4.08 & $1.68 \mathrm{e}-10$ & 4.05 & $2.49 \mathrm{e}-10$ & 4.04 \\
\hline $1 / 512$ & $1 / 512$ & $5.62 \mathrm{e}-12$ & 3.79 & $6.23 e-12$ & 4.08 & $1.01 \mathrm{e}-11$ & 4.05 & $1.56 \mathrm{e}-11$ & 4.00 \\
\hline
\end{tabular}
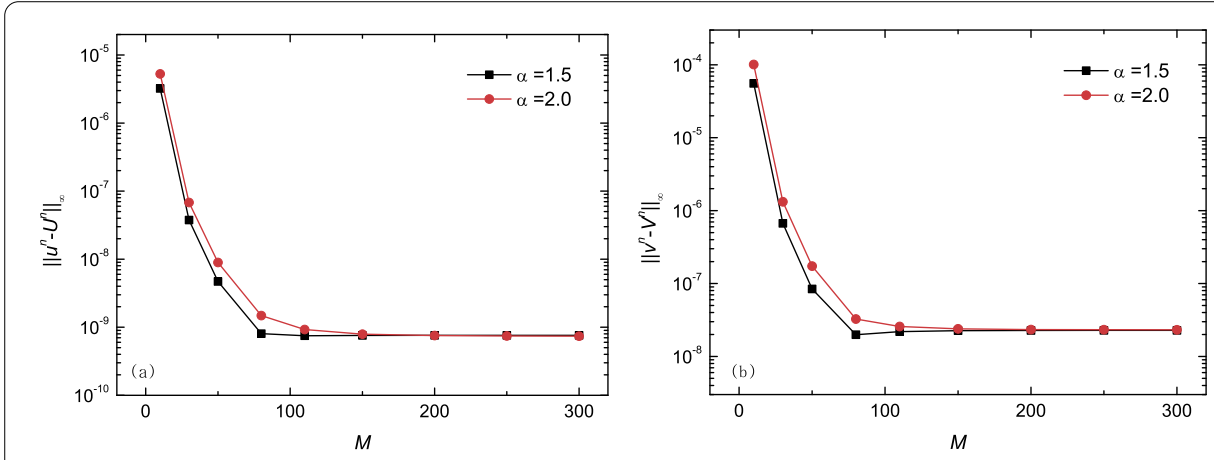

Figure 1 Numerical errors obtained by the fourth-order method for fixed $\tau=0.001$.(a) Numerical error of $\widetilde{U}$ at $T=1$;(b) Numerical error of $\widetilde{V}$ at $T=1$

Example 2 In this test, we take the following parameters:

$$
\begin{aligned}
& v_{1}=0.3, \quad \eta_{1}=0.5, \quad \kappa_{1}=-\frac{v_{1}\left(3 \sqrt{1+4 v_{1}^{2}}-1\right)}{2\left(2+9 v_{1}^{2}\right)}, \\
& \zeta_{1}=-1, \quad \delta_{1}=\kappa_{1}, \quad \beta_{1}=\zeta_{1}, \quad \gamma_{1}=0, \\
& v_{2}=0.3, \quad \eta_{2}=0.6, \quad \kappa_{2}=-\frac{v_{2}\left(3 \sqrt{1+4 v_{2}^{2}}-1\right)}{2\left(2+9 v_{2}^{2}\right)}, \\
& \zeta_{2}=-1, \quad \delta_{2}=\kappa_{2}, \quad \beta_{2}=\zeta_{2}, \quad \gamma_{2}=0 .
\end{aligned}
$$

In the computation, we use our proposed fourth-order method, where the computational interval is chosen as $[-15,15]$, final time is set to be $T=1$, and the initial value is taken as

$$
u(x, 0)=\operatorname{sech}(x) e^{2 i x}, \quad v(x, 0)=\operatorname{sech}(x) e^{2 i x} .
$$

The "exact solution" is computed on the very fine mesh $h=1 / 256, \tau=1 / 256$.

Table 3 and Table 4 list the errors and the convergence orders for the method with $\alpha=$ $1.2,1.5,1.8,2.0$ in the $L^{\infty}$-norm. And these results confirm the fourth-order convergence both in time and space variables.

\section{Conclusion}

In this paper, we developed a fourth-order linearized implicit finite difference method for the CFGLE. The method is unconditionally stable. Moreover, a rigorous analysis of the 
Table $3 L^{\infty}$-norm errors and convergence orders of $\tilde{U}$ obtained by the fourth-order scheme for Example 2

\begin{tabular}{|c|c|c|c|c|c|c|c|c|c|}
\hline \multirow[t]{2}{*}{$\bar{\tau}$} & \multirow[t]{2}{*}{$h$} & \multicolumn{2}{|l|}{$\alpha=1.2$} & \multicolumn{2}{|l|}{$\alpha=1.5$} & \multicolumn{2}{|l|}{$\alpha=1.8$} & \multicolumn{2}{|l|}{$\alpha=2.0$} \\
\hline & & $\left\|u^{n}-\tilde{U}^{n}\right\|_{\infty}$ & Order & $\left\|u^{n}-\widetilde{U}^{n}\right\|_{\infty}$ & Order & $\left\|u^{n}-\tilde{U}^{n}\right\|_{\infty}$ & Order & $\left\|u^{n}-\widetilde{U}^{n}\right\|_{\infty}$ & Order \\
\hline $1 / 8$ & $1 / 8$ & $5.65 e-02$ & - & $2.22 \mathrm{e}-02$ & - & $2.58 \mathrm{e}-02$ & - & $2.15 \mathrm{e}-02$ & - \\
\hline $1 / 16$ & $1 / 16$ & $8.41 \mathrm{e}-03$ & 2.75 & $3.32 \mathrm{e}-03$ & 2.74 & $2.17 \mathrm{e}-03$ & 3.57 & $1.46 \mathrm{e}-03$ & 3.88 \\
\hline $1 / 32$ & $1 / 32$ & $7.56 \mathrm{e}-04$ & 3.47 & $2.86 \mathrm{e}-04$ & 3.54 & $1.43 e-04$ & 3.92 & $9.45 e-05$ & 3.95 \\
\hline $1 / 64$ & $1 / 64$ & $5.35 \mathrm{e}-05$ & 3.82 & $1.93 e-05$ & 3.89 & $9.18 \mathrm{e}-06$ & 3.96 & $6.11 \mathrm{e}-06$ & 3.95 \\
\hline
\end{tabular}

Table $4 L^{\infty}$-norm errors and convergence orders of $\widetilde{V}$ obtained by the fourth-order scheme for Example 2

\begin{tabular}{|c|c|c|c|c|c|c|c|c|c|}
\hline \multirow[t]{2}{*}{$\tau$} & \multirow[t]{2}{*}{$h$} & \multicolumn{2}{|l|}{$\alpha=1.2$} & \multicolumn{2}{|l|}{$\alpha=1.5$} & \multicolumn{2}{|l|}{$\alpha=1.8$} & \multicolumn{2}{|l|}{$\alpha=2.0$} \\
\hline & & $\left\|v^{n}-\widetilde{V}^{n}\right\|_{\infty}$ & Order & $\left\|v^{n}-\widetilde{V}^{n}\right\|_{\infty}$ & Order & $\left\|v^{n}-\widetilde{V}^{n}\right\|_{\infty}$ & Order & $\left\|v^{n}-\widetilde{V}^{n}\right\|_{\infty}$ & Order \\
\hline $1 / 8$ & $1 / 8$ & $4.91 \mathrm{e}-02$ & 1.55 & $2.13 \mathrm{e}-02$ & 2.45 & $2.62 \mathrm{e}-02$ & 2.41 & $2.14 \mathrm{e}-02$ & 2.86 \\
\hline $1 / 16$ & $1 / 16$ & $7.69 e-03$ & 2.67 & $3.27 \mathrm{e}-03$ & 2.71 & $2.14 \mathrm{e}-03$ & 3.62 & $1.41 \mathrm{e}-03$ & 3.93 \\
\hline $1 / 32$ & $1 / 32$ & $7.16 \mathrm{e}-04$ & 3.43 & $2.83 e-04$ & 3.53 & $1.40 \mathrm{e}-04$ & 3.93 & $9.17 \mathrm{e}-05$ & 3.94 \\
\hline $1 / 64$ & $1 / 64$ & $5.15 e-05$ & 3.80 & $1.92 \mathrm{e}-05$ & 3.88 & $8.98 \mathrm{e}-06$ & 3.97 & $5.92 \mathrm{e}-06$ & 3.95 \\
\hline
\end{tabular}

proposed difference scheme is carried out, which includes the unconditional stability and the $L^{\infty}$-norm convergence of the method. Moreover, Richardson extrapolation is used to increase the temporal accuracy to fourth order. Numerical tests are performed to validate our theoretical findings.

This paper only focuses on the coupled space fractional Ginzburg-Landau equations, where time delay is ignored. As is well known, time delay has been receiving considerable attention and eliciting widespread interest [33-37]. However, the coupled space fractional Ginzburg-Landau equations is a nonlinear system, the convergence analysis for the timedelay case is not a matter of standard error analysis. We leave it as the future work.

\section{Acknowledgements}

The authors would like to acknowledge partial financial support from the State High-Tech Development Plan of China under Grant 2018YFC0603202 and the National Science Foundation of China under Grant 41830107, Grant 41811530010, and Grant 41574120.

\section{Funding}

This work was supported in part by the State High-Tech Development Plan of China under Grant 2018 YFC0603202 and in part by the National Science Foundation of China under Grant 41830107, Grant 41811530010, and Grant 41574120.

\section{Competing interests}

All authors declare that there is no conflict of interest regarding the publication of this paper.

\section{Authors' contributions}

All authors contributed equally to the writing of this paper. All authors read and approved the final paper.

\section{Publisher's Note}

Springer Nature remains neutral with regard to jurisdictional claims in published maps and institutional affiliations.

Received: 16 August 2019 Accepted: 18 October 2019 Published online: 28 October 2019

\section{References}

1. Aranson, I.S., Kramer, L.: The world of the complex Ginzburg-Landau equation. Rev. Mod. Phys. 74, 99-143 (2002)

2. Tarasov, V., Zaslavsky, G.: Fractional Ginzburg-Landau equation for fractal media. Physica A 354, 249-261 (2005)

3. Tarasov, V., Zaslavsky, G.: Fractional dynamics of coupled oscillators with long-range interaction. Chaos 16, 023110 (2006)

4. Milovanov, A., Rasmussen, J.: Fractional generalization of the Ginzburg-Landau equation: an unconventional approach to critical phenomena in complex media. Phys. Lett. A 337, 75-80 (2005) 
5. Mvogo, A., Tambue, A., et al.: Localized numerical impulse solutions in diffuse neural networks modeled by the complex fractional Ginzburg-Landau equation. Commun. Nonlinear Sci. Numer. Simul. 39, 396-410 (2016)

6. Shu, J., Li, P., et al.: Random attractors for the stochastic coupled fractional Ginzburg-Landau equation with additive noise. J. Math. Phys. 56, 102702 (2015)

7. Lord, G.J:: Attractors and inertial manifolds for finite-difference approximations of the complex Ginzburg-Landau equation. SIAM J. Numer. Anal. 34, 1483-1512 (1997)

8. Xu, Q., Chang, Q.: Difference methods for computing the Ginzburg-Landau equation in two dimensions. Numer. Methods Partial Differ. Equ. 27, 507-528 (2011)

9. Wang, T., Guo, B:: Analysis of some finite difference schemes for two-dimensional Ginzburg-Landau equation. Numer. Methods Partial Differ. Equ. 27, 1340-1363 (2011)

10. Zhang, Y., Sun, Z., Wang, T.: Convergence analysis of a linearized Crank-Nicolson scheme for the two-dimensional complex Ginzburg-Landau equation. Numer. Methods Partial Differ. Equ. 29, 1487-1503 (2013)

11. Hao, Z., Sun, Z., Cao, W.: A three-level linearized compact difference scheme for the Ginzburg-Landau equation. Numer. Methods Partial Differ. Equ. 31, 876-899 (2015)

12. Wang, P., Huang, C.: An implicit midpoint difference scheme for the fractional Ginzburg-Landau equation. J. Comput. Phys. 312, 31-49 (2016)

13. Hao, Z., Sun, Z:: A linearized high-order difference scheme for the fractional Ginzburg-Landau equation. Numer. Methods Partial Differ. Equ. 33, 105-124 (2017)

14. He, D., Pan, K.: An unconditionally stable linearized difference scheme for the fractional Ginzburg-Landau equation. Numer. Algorithms 79, 899-925 (2018)

15. Wang, P., Huang, C.: An efficient fourth-order in space difference scheme for the nonlinear fractional Ginzburg-Landau equation. BIT Numer. Math. 58, 783-805 (2018)

16. Li, M., Huang, C.: An efficient difference scheme for the coupled nonlinear fractional Ginzburg-Landau equations with the fractional Laplacian. Numer. Methods Partial Differ. Equ. 35, 394-421 (2019)

17. Zhao, X., Sun, Z., Hao, Z.: A fourth-order compact ADI scheme for two-dimensional nonlinear space fractional Schrödinger equation. SIAM J. Sci. Comput. 36, A2865-A2886 (2014)

18. Yang, Q., Liu, F., Turner, I.: Numerical methods for fractional partial differential equations with Riesz space fractional derivatives. Appl. Math. Model. 34, 200-218 (2010)

19. Wang, D., Xiao, A., Yang, W.: Crank-Nicolson difference scheme for the coupled nonlinear Schrödinger equations with the Riesz space fractional derivative. J. Comput. Phys. 242, 670-681 (2013)

20. Li, M., Gu, X., et al.: A fast linearized conservative finite element method for the strongly coupled nonlinear fractiona Schrödinger equations. J. Comput. Phys. 358, 256-282 (2018)

21. Podlubny, l.: Fractional Differential Equations. Academic Press, San Diego (1999)

22. Kilbas, A., Srivastava, H., Trujillo, J.: Theory and Applications of Fractional Differential Equations. Elsevier, Amsterdam (2006)

23. Ortigueira, M.: Riesz potential operators and inverses via fractional centred derivatives. Int. J. Math. Math. Sci. 2006 Article ID 48391 (2006)

24. Celik, C., Duman, M.: Crank-Nicolson method for the fractional diffusion equation with the Riesz fractional derivative. J. Comput. Phys. 231, 1743-1750 (2012)

25. Wang, D., Xiao, A., Yang, W.: Maximum-norm error analysis of a difference scheme for the space fractional CNLS. Appl. Math. Comput. 257, 241-251 (2015)

26. He, D., Pan, K.: An unconditionally stable linearized CCD-ADI method for generalized nonlinear Schrödinger equations with variable coefficients in two and three dimensions. Comput. Math. Appl. 73, 2360-2374 (2017)

27. He, D., Pan, K., Yue, X.: A positivity preserving and free energy dissipative difference scheme for the Poisson-Nernst-Planck system. J. Sci. Comput. 81, 436-458 (2019)

28. He, D., Pan, K.: Maximum norm error analysis of an unconditionally stable semi-implicit scheme for multi-dimensional Allen-Cahn equations. Numer. Methods Partial Differ. Equ. 35, 955-975 (2019)

29. Cai, W., He, D., Pan, K.: A linearized energy-conservative finite element method for the nonlinear Schrödinger equation with wave operator. Appl. Numer. Math. 140, 183-198 (2019)

30. Wang, P., Huang, C.: An energy conservative difference scheme for the nonlinear fractional Schrödinger equations J. Comput. Phys. 293, 238-251 (2015)

31. Holte, J.M.: Discrete Gronwall lemma and applications. In: MAA-NCS Meeting at the University of North Dakota, 24 October 2009, pp. 1-7 (2009)

32. He, D., Pan, K.: A linearly implicit conservative difference scheme for the generalized Rosenau-Kawahara-RLW equation. Appl. Math. Comput. 271, 323-336 (2015)

33. Xu, L., Sam Ge, S.: Asymptotic behavior analysis of complex-valued impulsive differential systems with time-varying delays. Nonlinear Anal. Hybrid Syst. 27, 13-28 (2018)

34. Xu, L., Dai, Z., He, D.: Exponential ultimate boundedness of impulsive stochastic delay differential equations. Appl. Math. Lett. 85, 70-76 (2018)

35. $\mathrm{Xu}, \mathrm{L} ., \mathrm{Chu}, \mathrm{X} ., \mathrm{Hu}, \mathrm{H}$. : Exponential ultimate boundedness of non-autonomous fractional differential systems with time delay and impulses. Appl. Math. Lett. 99, 106000 (2020)

36. Morgado, M.L., Ford, N.J., Lima, P.M.: Analysis and numerical methods for fractional differential equations with delay J. Comput. Appl. Math. 252, 159-168 (2013)

37. An, T.V., Vu, H., Hoa, N.V.: A new technique to solve the initial value problems for fractional fuzzy delay differential equations. Adv. Differ. Equ. 2017, 181 (2017) 